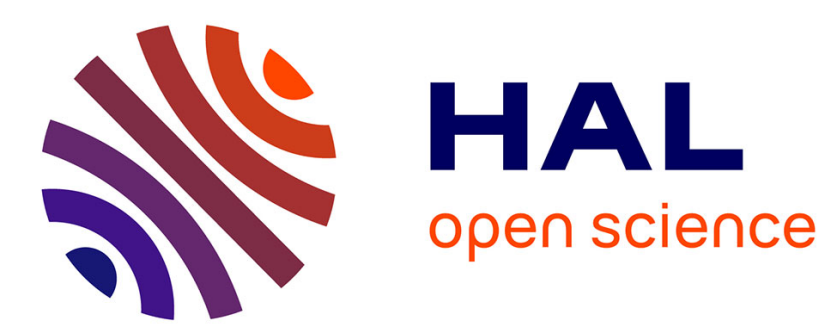

\title{
Directed polymers in a random medium : 1/d expansion and the n-tree approximation
}

\author{
J. Cook, Bernard Derrida
}

\section{To cite this version:}

J. Cook, Bernard Derrida. Directed polymers in a random medium: 1/d expansion and the n-tree approximation. Journal of Physics A: Mathematical and General (1975 - 2006), 1990, 23 (9), pp.15231554. 10.1088/0305-4470/23/9/017 . hal-03282951

\section{HAL Id: hal-03282951 \\ https://hal.science/hal-03282951}

Submitted on 19 Jul 2021

HAL is a multi-disciplinary open access archive for the deposit and dissemination of scientific research documents, whether they are published or not. The documents may come from teaching and research institutions in France or abroad, or from public or private research centers.
L'archive ouverte pluridisciplinaire HAL, est destinée au dépôt et à la diffusion de documents scientifiques de niveau recherche, publiés ou non, émanant des établissements d'enseignement et de recherche français ou étrangers, des laboratoires publics ou privés. 


\title{
Directed polymers in a random medium: $1 / d$ expansion and the $n$-tree approximation
}

\author{
J Cook $\dagger \ddagger$ and B Derrida $\dagger$ \\ † Service de Physique Théorique§ de Saclay, 91191 Gif-sur-Yvette Cedex, France \\ $\ddagger$ Department of Physics, Edinburgh University, Edinburgh EH9 3JZ, UK
}

Received 25 October 1989

\begin{abstract}
We develop a method which allows one to obtain 1/d expansions for the problem of directed polymers in a random medium. Using this method, we expand thermal properties (such as the free energy, the specific heat), the overlaps and the fluctuations of the transverse displacement. Our results are consistent with the existence of a finite upper critical dimension above which the low-temperature phase is mean field like, i.e. characterised by a broad distribution of overlaps (broken replica symmetry) and transverse fluctuations which scale as the length of the polymer.
\end{abstract}

\section{Introduction}

The problem of directed polymers in a random medium can be formulated in its simplest lattice version as follows (Kardar 1987, Zhang 1987, Kardar and Zhang 1987, Cook and Derrida 1989b). For each bond, $i j$, of some regular lattice one chooses a random energy, $\varepsilon_{i j}$, according to a given probability distribution $\rho\left(\varepsilon_{i j}\right)$. One then considers all directed walks of length $L$ starting at a fixed origin on the lattice. A directed walk is, by definition, one stretched along a single longitudinal direction, with fluctuations in the transverse directions only. In this paper we shall consider walks on a $d$-dimensional hypercubic lattice, directed along the $(1,1, \ldots, 1)$ direction of the lattice. This means that at each step one coordinate must increase by one, so that if the walks commence at $\boldsymbol{r}=\left(x_{1}, \ldots, x_{d}\right)$ they can end at any point $\boldsymbol{r}^{\prime}=$ $\left(x_{1}+n_{1}, \ldots, x_{d}+n_{d}\right)$ with $n_{1}+\ldots+n_{d}=L$. The energy, $E_{w}$, of a walk, $w$, is defined as

$$
E_{w}=\sum_{i j \in w} \varepsilon_{i j}
$$

where the summation runs over all the bonds, $i j$, visited by the walk $w$.

Three kinds of properties can be studied for such a system: thermal properties, overlaps and geometrical properties.

(i) Thermal properties. These can be obtained from the partition function $Z$ :

$$
Z_{L}(\boldsymbol{r})=\sum_{w} \exp \left(-E_{w} / T\right) \text {. }
$$

In (2) the sum includes all the directed walks of length $L$ starting from the point $r$, and $T$ is the temperature. From the knowledge of $Z$, one can calculate the free energy, the energy and the specific heat as functions of the temperature and then study the 
phase diagram. In the thermodynamic limit $(L \rightarrow \infty)$, one can observe phase transitions between a high-temperature phase where the effect of disorder is weak and a lowtemperature phase very similar to a spin-glass phase.

At zero temperature, the problem becomes an optimisation problem: the problem of finding the walk of lowest energy. In addition to the value of the ground-state energy, one can study its fluctuations (Zhang 1987, Kardar and Zhang 1987, Huse et al 1985) which are characterised by an exponent $\omega$ which depends on the dimension of the lattice

$$
\left\langle E_{G S}^{2}\right\rangle-\left\langle E_{G S}\right\rangle^{2} \sim L^{2 \omega}
$$

where \langle\rangle means an average over disorder.

(ii) Overlaps. As one can think of the low-temperature phase as a phase where the directed walks get trapped in deep valleys, it is useful, in order to study the statistical properties of the landscape, to define the overlap between two walks and the probability distribution of these overlaps (Derrida and Spohn 1988, Cook and Derrida 1989b). This overlap is defined by the fraction of their length that the two walks spend together.

(iii) Geometrical properties. The effect of disorder is to influence the transverse fluctuations of the directed walks. These can be characterised by an exponent $v$ :

$$
Z^{-1} \sum_{w^{\prime}}(\boldsymbol{R}(w))^{2} \exp \left(-E_{w} / T\right) \sim L^{2 v}
$$

where $\boldsymbol{R}(w)$ is the projection of the walk on the transverse directions.

The problem of directed polymers in a random medium has close analogies with two other problems in statistical mechanics: growth models and spin glasses.

The link with models of growing surfaces (Eden model or ballistic deposition) has been discussed by Kardar and Zhang (1987) and McKane and Moore (1988). They showed that the Kardar-Parisi-Zhang equation (Kardar et al 1986) for growth models could be interpreted as a continuous version of the directed polymer problem. This mapping allows one to relate the exponents $\omega$ and $\nu$ defined by (3) and (4) to the critical exponents characterising the fluctuations and roughness of the growing interface (Krug and Spohn 1988, Wolf and Kertész 1987, Kim and Kosterlitz 1989).

The link with spin glasses has been made in the mean-field limit (Derrida and Spohn 1988). It was shown in the mean-field theory of the directed polymer problem that the low-temperature phase is characterised by a broad distribution of overlaps as in Parisi's mean-field theory of spin glasses (Parisi 1980a, b, Mezard et al 1987). For spin glasses, the relevance of the Parisi solution in finite dimension has been a topic of controversy for the past decade (Sourlas 1986, Huse and Fisher 1987, 1988). One difficulty in trying to extend the Parisi solution to finite-dimensional systems is that the mean-field free energy is sufficiently complicated to make expansions around it technically very difficult (Bray and Moore 1979, de Dominicis and Kondor 1985). As the analytic expression for the free energy of the low-temperature phase is much simpler in the polymer problem, it should be an easier system in which to test the validity of the Parisi approach in finite-dimensional systems (Cook and Derrida 1989a).

Let us now summarise what is already known about the problem of directed polymers in a random medium.

A phase transition is known to exist for $d>2+1$ (two transverse plus one longitudinal directions) (Imbrie and Spencer 1988). Above the transition temperature, $T_{c}$, the thermal properties take simple forms, the annealed and quenched free energies being the same. In the mean-field limit (Derrida and Spohn 1988) one can calculate 
the transition temperature, whereas for finite-dimensional systems only bounds have been obtained (Cook and Derrida 1989b).

A lot of work has been done on determining the exponents $\omega$ and $\nu$ (see (3), (4)) at low temperature. In $1+1$ dimensions the problem can be mapped on to a Burgers equation and a renormalisation analysis gives $\omega=\frac{1}{3}$ and $\nu=\frac{2}{3}$ (Forster et al 1977, Huse et al 1985). Little is known analytically about the exponents in higher dimension. A recent renormalisation group calculation (Halpin-Healy 1989, Nattermann and Renz 1988) has indicated a finite upper critical dimension $d_{\mathrm{c}}=4+1$. However, numerical simulations carried out by evaluating the surface scaling exponents for growth models have led to different conjectures. Wolf and Kertész (1987) suggest $\omega=1 /(2 d-1)$ and $\nu=d /(2 d-1)$ whilst Kim and Kosterlitz (1989) propose $\omega=1 /(d+1)$ and $\nu=$ $(d+2) / 2(d+1)$ both giving no finite upper critical dimension. Recent simulations by Renz (1989) are intermediate between these two conjectures.

It is currently not established whether the exponents $\omega$ and $\nu$ vary continuously with dimension up to $d=\infty$ or whether they take their mean-field values, $\omega=0$ and $\nu=\frac{1}{2}$, above a certain finite dimension $d_{\mathrm{c}}$. In all cases, one expects these exponents to be related by a simple relation $\omega+1=2 \nu$ (Krug 1987).

Having summarised the problem and some of the results known already, let us now outline the rest of the paper and the new results we shall present. Firstly we shall discuss the mean-field version of the problem, recalling the methods of solution and the main results. Then, in section 3 we present the $1 / d$ expansion method, first introduced in a previous work (Cook and Derrida 1989a), which is based on a series of approximations which we call ' $n$-tree' approximations. This will allow us to determine the $1 / d$ expansion of the free energy and of the ground-state energy when the distribution $\rho(\varepsilon)$ of energies is exponential. We extend the method to obtain the fluctuations of the transverse displacement in section 4 and we calculate the overlap in section 5 . In section 6 we discuss how the results would be modified by considering other distributions of disorder. Lastly in section 7 we test our expansion method (based on the $n$-tree approximation) on several problems which can be solved by alternative approaches.

\section{The tree problem}

A mean-field version of the problem of directed polymers in a random medium can be solved exactly (Derrida and Spohn 1988), for any distribution of disorder, by taking the regular lattice to be a branch of a tree (see figure 1). This problem can be solved by several methods: an analogy with travelling waves, a replica approach or by using results known on the GREM (Derrida and Gardner 1986). In this section we shall outline the travelling wave approach and present the main mean-field results. The replica approach is described in appendix 1.

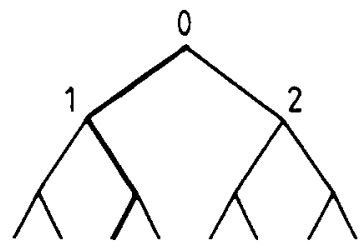

Figure 1. Directed walk on a tree with $K=2$. 
Consider the branch of a tree, with coordination number, $K+1$ (figure 1). For each bond $i j$ of the lattice one chooses a random energy, $\varepsilon_{i j}$, according to a given distribution $\rho\left(\varepsilon_{i j}\right)$. If $Z_{L}(0)$ is the partition function of the $K^{L}$ directed walks of length $L$ starting at 0 , defined by (1) and (2), one can write a recursion relation connecting $Z_{L}$ and $Z_{L+1}$ :

$$
Z_{L+1}(0)=\sum_{i=1}^{K} \exp \left(-\varepsilon_{0 i} / T\right) Z_{L}(i)
$$

where $Z_{L}(i)$ is the partition function of the walks in the $i$ th branch and $\varepsilon_{0 i}$ is the energy of the bond connecting the origin to the $i$ th branch. The energies $\varepsilon_{i j}$ are independent random variables and so the partition function itself is random. It is therefore natural to consider a generating function of $Z_{L}, H_{L}(x)$ defined by

$$
H_{L}(x)=\left\langle\exp \left[-\exp (-x / T) Z_{L}\right]\right\rangle .
$$

Using $H_{L}(x)$ the recursion (5) takes the simple form

$$
H_{L+1}(x)=\left(\int \rho(\varepsilon) H_{L}(x+\varepsilon) \mathrm{d} \varepsilon\right)^{\kappa}
$$

where we have used the fact that $\varepsilon_{01}, \ldots, \varepsilon_{0 K}$ and $Z_{L}(1), \ldots, Z_{L}(K)$ are independent random variables. Notice that $(7)$ is independent of temperature. The temperature dependence only remains in the initial condition (8).

$$
H_{0}(x)=\exp [-\exp (-x / T)]
$$

It can easily be seen from (6) and (8) that

$$
H_{L}(x) \rightarrow \begin{cases}1 & \text { when } x \rightarrow \infty \\ 0 & \text { when } x \rightarrow-\infty\end{cases}
$$

Hence $H_{L}(x)$ has the shape of a wavefront. Equation (7) is a travelling wave equation, belonging to a class of nonlinear equations of the diffusion-reaction type, and has properties similar to those of the KPP (Kolmogorov-Petrovsky-Piscounov) equation (Bramson 1983)

$$
\frac{\partial H}{\partial L}=\frac{\partial^{2} H}{\partial x^{2}}+H^{2}-H
$$

Finding, for large $L$, the solution of the travelling wave equation (7) or (10) is not simple. However, it has been shown by Hammersley (1974) for (7) and by Bramson (1983) for (10), that for large $L$, the solutions of these equations become travelling waves, $W$, which move with velocity $v$

$$
H_{L}(x)=W(x-v L-c(L))
$$

where $c(L) / L \rightarrow 0$ as $L \rightarrow \infty$.

In general the exact form of $W$ is not known but the analytic expression of the velocity is easy to obtain. The velocity of the wave, $v$, depends upon the initial condition. For an initial condition of the form

$$
H_{0}(x)= \begin{cases}1-\mathrm{e}^{-\gamma x} & x \rightarrow \infty \\ 0 & x \rightarrow-\infty\end{cases}
$$


with $H_{0}(x)$ a monotonic function, it has been shown (Hammersley 1974, Bramson 1983) that the velocity is given by

$$
v= \begin{cases}G_{1}(\gamma) & \text { if } \gamma \leqslant \gamma_{\min } \\ G_{1}\left(\gamma_{\min }\right)=v_{\min } & \text { if } \gamma \geqslant \gamma_{\min }\end{cases}
$$

where

$$
G_{1}(\gamma)=\frac{1}{\gamma} \log \left(K \int \rho(\varepsilon) \mathrm{e}^{-\gamma \varepsilon} \mathrm{d} \varepsilon\right)
$$

and $\gamma_{\min }$ is the value of $\gamma$ that minimises the velocity, i.e.

$$
\left.\frac{\mathrm{d}}{\mathrm{d} \gamma} G_{1}(\gamma)\right|_{\gamma_{\min }}=0
$$

( $G_{1}(\gamma)$ has in general a single minimum: this could be shown by using the fact that $\gamma G_{1}(\gamma)$ is always a convex function of $\left.\gamma\right)$. From (13) we see that the exponential decay, $\gamma$, of the initial condition controls the velocity of the travelling waves. If $\gamma<\gamma_{\text {min }}$ the velocity varies continuously with $\gamma$, whereas if $\gamma \geqslant \gamma_{\min }$ the velocity remains fixed at its minimal value, $v_{\min }$.

These properties of the travelling waves $((11)-(14))$ can be mapped onto properties of the partition function. Because of (6), the distribution of $\log Z_{L}$ is concentrated near the place where the wavefront is located. This implies from (11) that $-v$ can be identified with the free energy per unit length of the sytem, so we have

$$
\lim _{L \rightarrow \infty} \frac{T}{L}\left\langle\log Z_{L}\right\rangle= \begin{cases}G_{1}(1 / T) & T \geqslant T_{\mathrm{c}} \\ G_{1}\left(\gamma_{\min }\right) & T \leqslant T_{\mathrm{c}}\end{cases}
$$

where

$$
T_{\mathrm{c}} \gamma_{\min }=1
$$

This result is the same as that obtained by the replica method (equation (A1.12) of appendix 1). From (15) one sees that the entropy vanishes in the whole of the low-temperature phase, so that the system is completely frozen. One can conclude from (11) that the width of the probability distribution of $\log Z_{L}$ must be of order one, and this means that the energy fluctuation exponent, defined by (3), $\omega=0$. It is not, however, possible to obtain an explicit expression for the ground-state energy fluctuations as this would require the knowledge of the shape of the travelling wave.

Another set of quantities which are of interest in this problem are the overlaps. If one defines $q_{2}\left(w, w^{\prime}\right)$ to be the fraction of their length that two walks $w$ and $w^{\prime}$, of length $L$, spend together, one can define the average overlap, $\left\langle q_{2}\right\rangle$, to be

$$
\left\langle q_{2}\right\rangle=\left\langle\frac{1}{Z_{L}^{2}} \sum_{w} \sum_{w^{\prime}} q_{2}\left(w, w^{\prime}\right) \exp \left(-\frac{E_{w^{\prime}}}{T}-\frac{E_{w^{\prime}}}{T}\right)\right\rangle .
$$

Similarly one can define the average overlap between $m$ walks, $\left\langle q_{m}\right\rangle$. These overlaps are analogous to the overlaps which occur in the mean-field spin glass (Parisi 1983, Mezard et al 1984). Often it is useful to consider the probability distribution of the overlaps, $P_{m}(q)$. When replica symmetry breaking occurs one expects a many-valley landscape, in which the overlaps, $q_{m}$, will not be self-averaging. $P_{2}(q)$ will then be a non-trivial distribution function, which we define as

$$
P_{2}(q)=\left\langle\frac{1}{Z_{L}^{2}} \sum_{w^{\prime}} \sum_{w^{\prime}} \delta\left(q-q_{2}\left(w, w^{\prime}\right)\right) \exp \left(-\frac{E_{w}}{T}-\frac{E_{w^{*}}}{T}\right)\right\rangle
$$


with similar formulae pertaining for $P_{m}(q)$. In appendix 1, a replica approach is used to show, along with results (13)-(16), that the average overlap, $\left\langle q_{2}\right\rangle$, and the distribution $P_{2}(q)$ are given by

$$
\left\langle q_{2}\right\rangle= \begin{cases}1-T / T_{\mathrm{c}} & T \leqslant T_{\mathrm{c}} \\ 0 & T \geqslant T_{\mathrm{c}}\end{cases}
$$

and

$$
P_{2}(q)= \begin{cases}\frac{T}{T_{\mathrm{c}}} \delta(q)+\left(1-\frac{T}{T_{\mathrm{c}}}\right) \delta(q-1) & T \leqslant T_{\mathrm{c}} \\ \delta(q) & T \geqslant T_{\mathrm{c}}\end{cases}
$$

for the tree problem (Derrida and Spohn 1988).

The fact that $P_{2}(q)$ is not a single delta function for $T<T_{\mathrm{c}}$ indicates a free energy landscape with a many-valley structure. Two walks lying in the same valley have an overlap $q_{2}=1$, whereas walks lying in different valleys have zero overlap.

To summarise, we have seen in this section that a travelling wave approach can be used to solve the mean-field problem of polymers in random media. The main results (13)-(16), (19), (20) for the mean-field limit will provide the basis for the $1 / d$ expansions which we shall now present in sections 3,4 and 5 .

\section{The $1 / d$ expansion and the $n$-tree approximation}

In this section we present the method of ' $n$-tree' approximations and show how this can be used to develop a $1 / d$ expansion for the free energy of the directed polymer on a hypercubic lattice (Cook and Derrida 1989a). We use the expansion to derive formulae for the free energy, ground-state energy and specific heat in the lowtemperature phase (equations (42), (46) and (44), respectively) and the transition temperature (equation (45)).

Consider the problem of directed polymers on a hypercubic lattice of dimension $d$. As usual one chooses a random energy, $\varepsilon_{i j}$, according to a given probability distribution, $\rho(\varepsilon)$, for each bond $i j$ of the lattice. In this section and in sections 4 and 5 we shall choose (Dhar 1988)

$$
\rho(\varepsilon)=\exp (-\varepsilon) \quad \varepsilon \geqslant 0 .
$$

This choice will make the calculation of several integrals we shall require much simpler. The use of other distributions will be discussed in section 6 . Consider walks of length $L$, directed so that at each step one coordinate increases by one. This means that one directs the walks along the $(1,1, \ldots, 1)$ direction, so that if the walks commence at $\boldsymbol{r}=\left(x_{1}, \ldots, x_{d}\right)$ they can end at any point $\boldsymbol{r}^{\prime}=\left(x_{1}+n_{1}, \ldots, x_{d}+n_{d}\right)$ with $n_{1}+\ldots+n_{d}=$ $L$. There are $d^{L}$ directed walks of length $L$ emanating from any given origin. The partition function, $Z_{L}(\boldsymbol{r})$, is defined by (1) and (2) as before.

One can write the following recursion for $Z_{L}(r)$ :

$$
Z_{L+1}(\boldsymbol{r})=\sum_{i=1}^{d} a_{i}(\boldsymbol{r}) Z_{L}\left(\boldsymbol{r}+\boldsymbol{e}_{i}\right)
$$

with

$$
a_{i}(\boldsymbol{r})=\exp \left(-\varepsilon_{r, r+e_{1}} / T\right)
$$


where $e_{i}$ are the unit vectors of the lattice in the directions in which a step is allowed, and the initial condition is

$$
Z_{0}(\boldsymbol{r})=1 \text {. }
$$

The recursion (22a) is very similar to the recursion (5) which was valid for the tree. The main difference is that, in $(22 a)$, the $Z_{L}\left(\boldsymbol{r}+\boldsymbol{e}_{i}\right)$ are correlated. For example $Z_{L}\left(\boldsymbol{r}+\boldsymbol{e}_{i}\right)$ and $Z_{L}\left(\boldsymbol{r}+\boldsymbol{e}_{j}\right)$ are correlated because they both depend on $Z_{L-1}\left(\boldsymbol{r}+\boldsymbol{e}_{i}+\boldsymbol{e}_{j}\right)$.

To cope with these correlations in a systematic way we have developed a series of approximations which we call ' $n$-tree' approximations. These become exact in the limit $n \rightarrow \infty$. To obtain the $n$-tree approximation to the problem, one iterates $(22 a) n$ times exactly and then neglects the remaining correlations. In other words one takes account of the correlations on the first $n$ steps of the lattice exactly and then constructs a tree from this motif, by neglecting other correlations. Diagrams of the 1-tree, 2-tree and 3-tree are shown for $d=2$ in figure 2 .

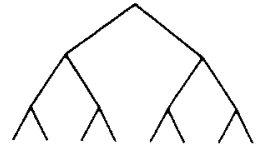

(a)

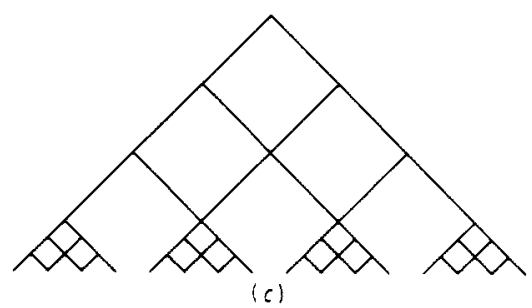

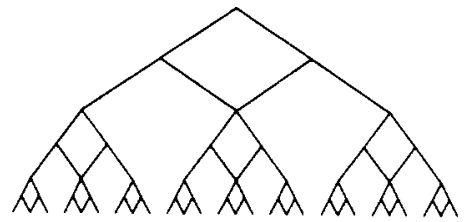

(b)

Figure 2. (a) 1-tree; (b) 2-tree; (c) 3-tree drawn for $d=2$.

\subsection{The 1-tree approximation}

The first approximation, a 1-tree, neglects all correlations on the right-hand side of (22a). This gives the mean-field problem of $(5)$, with $K=d$, the solution of which has been discussed in section 2. If we call the free energy per unit length of the $n$-tree $F_{n}(T)$, then from (13)-(16) we have

$$
F_{1}(T)= \begin{cases}-G_{1}(1 / T) & T \geqslant 1 / \gamma_{\min } \\ -G_{1}\left(\gamma_{\min }\right) & T \leqslant 1 / \gamma_{\min }\end{cases}
$$

where

$$
G_{1}(\gamma)=\frac{1}{\gamma} \log \left(d\left\langle\mathrm{e}^{-\gamma \digamma}\right\rangle\right)
$$

and $\gamma_{\min }$ is given by

$$
\left.\frac{\mathrm{d} G_{1}(\gamma)}{\mathrm{d} \gamma}\right|_{\gamma_{\min }}=0
$$


We use, as before, $\langle\ldots\rangle$ to denote an average over disorder, so here we have

$$
\left\langle\mathrm{e}^{-\gamma \varepsilon}\right\rangle \equiv \int \rho(\varepsilon) \mathrm{e}^{-\gamma \varepsilon} \mathrm{d} \varepsilon .
$$

For the distribution (21) one sees that

$$
G_{1}(\gamma)=\frac{1}{\gamma} \log \left(\frac{d}{\gamma+1}\right)
$$

If $d$ is large one can expand the free energy given by (24) and (26) in powers of $1 / d$, yielding

$$
F_{1}(T)= \begin{cases}\frac{1}{e d}+\frac{1}{e^{2} d^{2}}+\frac{3}{2 e^{3} d^{3}}+\mathrm{O}\left(d^{-4}\right) & T \leqslant T_{\mathrm{c}} \\ T \log [(1+T) / d T] & T \geqslant T_{\mathrm{c}}\end{cases}
$$

and one finds that the transition temperature, $T_{c}$, is given by

$$
T_{\mathrm{c}}=\frac{1}{\gamma_{\min }}=\frac{1}{e d}+\frac{2}{e^{2} d^{2}}+\frac{9}{2 e^{3} d^{3}}+\mathrm{O}\left(d^{-4}\right) .
$$

\subsection{The 2-tree approximation}

In the 1-tree approximation we have neglected all correlations in (22a). To extend the theory beyond the mean-field limit one must consider the correlations neglected so far. To start to do this we move to the 2-tree approximation. The 2-tree approximation is obtained by iterating $(22 a)$ twice to give

$$
\begin{aligned}
Z_{L+2}(\boldsymbol{r})=\sum_{i=1}^{d} & a_{i}(\boldsymbol{r}) a_{i}\left(\boldsymbol{r}+\boldsymbol{e}_{i}\right) Z_{L}\left(\boldsymbol{r}+2 \boldsymbol{e}_{i}\right) \\
& +\sum_{i=1}^{d-1} \sum_{j>i}^{d}\left[a_{i}(\boldsymbol{r}) a_{j}\left(\boldsymbol{r}+\boldsymbol{e}_{i}\right)+a_{j}(\boldsymbol{r}) a_{i}\left(\boldsymbol{r}+\boldsymbol{e}_{j}\right)\right] Z_{L}\left(\boldsymbol{r}+\boldsymbol{e}_{i}+\boldsymbol{e}_{j}\right)
\end{aligned}
$$

and now neglecting the correlations between the $Z_{L}$ on the RHS of (29). Again one ends up with a tree (see figure $2(b)$ ), but now it has $d+d(d-1) / 2$ branches rather than $d$ branches. The branches are of two types: $d$ branches have an energy which is the sum of two random energies $\varepsilon_{i}+\varepsilon_{j}$, whereas the other $d(d-1) / 2$ branches have an effective energy, $\varepsilon_{\text {eff }}$, given by

$$
\varepsilon_{\mathrm{eff}}=-T \log \left[a_{i}(\boldsymbol{r}) a_{j}\left(\boldsymbol{r}+\boldsymbol{e}_{i}\right)+a_{j}(\boldsymbol{r}) a_{i}\left(\boldsymbol{r}+\boldsymbol{e}_{j}\right)\right] .
$$

The effective energy on these $d(d-1) / 2$ branches depends upon the temperature and represents the fact that these branches are composed of two paths.

As a tree structure still remains the 2-tree can be solved exactly as before (equation (24)). If one defines

$$
\begin{aligned}
G_{2}(\gamma)=\frac{1}{2 \gamma} \log ( & d\left\langle\exp \left[-\gamma\left(\varepsilon_{1}+\varepsilon_{2}\right)\right]\right\rangle+\frac{1}{2} d(d-1) \\
\times & \left.\left.\times\left\{\exp \left[-\left(\varepsilon_{1}+\varepsilon_{2}\right) / T\right]+\exp \left[-\left(\varepsilon_{3}+\varepsilon_{4}\right) / T\right]\right\}^{\gamma T}\right\rangle\right)
\end{aligned}
$$


then the free energy per unit length of the 2-tree is given by

$$
F_{2}(T)= \begin{cases}-G_{2}\left(\frac{1}{T}\right) & T \geqslant T_{\mathrm{c}} \\ -G_{2}\left(\gamma_{\min }\right) & T \leqslant T_{\mathrm{c}}\end{cases}
$$

with $\gamma_{\min }$ the solution of

$$
\left.\frac{\mathrm{d} G_{2}(\gamma)}{\mathrm{d} \gamma}\right|_{\gamma_{\min }}=0
$$

The extra factor $\frac{1}{2}$ in (31) appears because when one iterates (29) $L$ times the polymer is of length $2 L$, not $L$. One sees that $\gamma_{\min }$ now depends upon the temperature, because the effective energy on $d(d-1) / 2$ of the branches was temperature dependent. $T_{\mathrm{c}}$ is still given by (16), i.e. is the solution of $T_{\mathrm{c}}=1 / \gamma_{\min }\left(T_{j l e O c}\right)$.

\subsection{The 3-tree approximation}

To get successively better approximations to the $d$-dimensional lattice one can consider in turn a 3-tree, 4-tree, etc. ... For example for the 3-tree

$$
G_{3}(\gamma)=\frac{1}{3 \gamma} \log \left[d\left(\mathrm{e}^{-\gamma \varepsilon}\right\rangle^{3}+d(d-1)\left\langle f_{2}(T)^{\gamma T}\right\rangle+\frac{1}{6} d(d-1)(d-2)\left\langle f_{3}(T)^{\gamma T}\right\rangle\right]
$$

where

$$
f_{2}(T)=a_{1} a_{2} a_{3}+a_{4} a_{5} a_{3}+a_{4} a_{6} a_{7}
$$

and

$$
f_{3}(T)=a_{1} a_{2} a_{7}+a_{1} a_{6} a_{10}+a_{4} a_{3} a_{7}+a_{4} a_{8} a_{11}+a_{5} a_{9} a_{10}+a_{5} a_{12} a_{11}
$$

where $a_{i}=\exp \left(-\varepsilon_{i} / T\right)$.

One can see that $f_{2}(T)$ and $f_{3}(T)$ are the partition functions associated with the directed walks linking the two marked points on diagrams 2 and 3 of figure 3 respectively. It is clear that as the number of energies that must be averaged over increases the problem becomes much harder. So, although the aim would be to proceed to successive $n$-tree approximations, the complexity of the calculations that would be involved makes this approach impossible. However, for large dimension the problem is tractable.

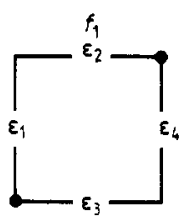

11)

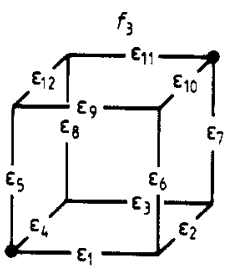

(3)

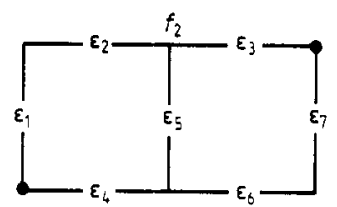

12)

Figure 3. $f_{1}$ is the sum of the weights of all the directed walks connecting the two marked points on the diagram $i$. 


\subsection{The 2-tree for large $d$}

When we expanded the tree solution for large $d$, equations $(27 a),(28)$, one saw that $T_{c}$ was of order $1 / d$. The key to obtaining the $1 / d$ expansion is to keep in mind that for large dimension $T_{\mathrm{c}}$ is small, of order $1 / d$, and $\gamma_{\min }$ is large, of order $d$.

It is the fact that the relevant temperatures are small $\sim 1 / d$ which simplifies the situation. To see this, consider (31). One needs to calculate

$$
\left\langle f_{1}(T)^{\gamma T}\right\rangle=\left\langle\left\{\exp \left[-\left(\varepsilon_{1}+\varepsilon_{2}\right) / T\right]+\exp \left[-\left(\varepsilon_{3}+\varepsilon_{4}\right) / T\right]\right\}^{\gamma T}\right\rangle
$$

corresponding to figure $3(a)$. As $T$ is at most of order $1 / d$ in the low-temperature phase, the leading contribution to (34) will result from the region in the space of $\varepsilon_{1}$, $\varepsilon_{2}, \varepsilon_{3}, \varepsilon_{4}$ where one of the two terms on the RHS of (34) is much larger than the other. One can rewrite (34) as

$$
\begin{aligned}
\left\langle f_{1}(T)^{\gamma T}\right\rangle=2\langle\exp (-\gamma \varepsilon)\rangle^{2}+\left(\left\langle\left\{\exp \left[-\left(\varepsilon_{1}+\varepsilon_{2}\right) / T\right]\right.\right.\right. \\
\left.\left.\left.+\exp \left[-\left(\varepsilon_{3}+\varepsilon_{4}\right) / T\right]\right\}^{\gamma T}\right\rangle-2(\exp (-\gamma \varepsilon)\rangle^{2}\right) .
\end{aligned}
$$

Using (21) one sees that the first term on the RHS of (35) is $2(\gamma+1)^{-2}$, whilst the second is only of order $\gamma^{-4}$. For example, it is easy to show that at $T=0$ the second term is $-2(3 \gamma+4)(\gamma+1)^{-2}(\gamma+2)^{-3}$. Using the rearrangement (35) one can recast $G_{2}(\gamma)$ in the form

$$
\begin{aligned}
G_{2}(\gamma)=\frac{1}{2 \gamma} \log \left[d^{2}\langle\exp (-\gamma \varepsilon)\rangle^{2}+\frac{1}{2} d(d-1)\right. \\
\left.\times\left(\left\langle\left\{\exp \left[-\left(\varepsilon_{1}+\varepsilon_{2}\right) / T\right]+\exp \left[-\left(\varepsilon_{3}+\varepsilon_{4}\right) / T\right]\right\}^{\gamma T}\right\rangle-2\langle\exp (-\gamma \varepsilon)\rangle^{2}\right)\right]
\end{aligned}
$$

\subsection{The 3-tree for large $d$}

To see how the high-dimension simplification works in a more complex case, consider $G_{3}(\gamma)$ given in (33). This was complicated because one had to evaluate $\left\langle f_{2}(T)^{\gamma T}\right\rangle$ and $\left\langle f_{3}(T)^{\gamma T}\right\rangle$. However, when $d$ is large these quantities simplify in the same way as $\left\langle f_{1}(T)^{\gamma T}\right\rangle$ in (35). Take first $\left\langle f_{2}(T)^{\gamma T}\right\rangle$ defined in $(33 b)$. As $T$ is small this is dominated by cases in which one of the three terms in $(33 b)$ is much larger than the other two. The next largest contribution comes from the situations when either the first pair or the last pair are much larger than the other term. Smaller again is the contribution due to cases when the first and last terms are of similar size, much greater than the middle term, and smallest of all is the effect of having all three terms of the same magnitude. Thus one can write

$$
\begin{aligned}
&\left\langle f_{2}(T)^{\gamma T}\right\rangle=3\left\langle a^{\gamma T}\right\rangle^{3}+2\left\langle a^{\gamma T}\right\rangle\left\{\left\langle\left(a_{1} a_{2}+a_{3} a_{4}\right)^{\gamma T}\right\rangle-2\left\langle a^{\gamma T}\right\rangle^{2}\right\} \\
&+\left\{\left\langle\left(a_{1} a_{2} a_{3}+a_{4} a_{5} a_{6}\right)^{\gamma T}\right\rangle-2\left(a^{\gamma T}\right\rangle^{3}\right\} \\
&+\left\{\left\langle\left(a_{1} a_{2} a_{3}+a_{4} a_{5} a_{3}+a_{4} a_{6} a_{7}\right)^{\gamma T}\right\rangle\right. \\
&-\left[\left\langle\left(a_{1} a_{2} a_{3}+a_{4} a_{5} a_{6}\right)^{\gamma T}\right\rangle-2\left\langle a^{\gamma T}\right\rangle^{3}\right] \\
&\left.-2\left\langle a^{\gamma T}\right\rangle\left[\left\langle\left(a_{1} a_{2}+a_{3} a_{4}\right)^{\gamma T}\right\rangle-2\left\langle a^{\gamma T}\right\rangle^{2}\right]-3\left\langle a^{\gamma T}\right\rangle^{3}\right\} .
\end{aligned}
$$

The leading term is now $3(\gamma+1)^{-3}$, using (21), the second term is of order $\gamma^{-5}$, the third term is of order $\gamma^{-6}$ and the last of order $\gamma^{-7}$. Similarly one can rewrite 
$\left\langle f_{3}(T)^{\gamma T}\right\rangle$ as

$$
\begin{aligned}
\left\langle f_{3}(T)^{\gamma T}\right\rangle=6 & \left\langle a^{\gamma T}\right\rangle^{3}+6\left\langle a^{\gamma T}\right\rangle\left\{\left\langle\left(a_{1} a_{2}+a_{3} a_{4}\right)^{\gamma T}\right\rangle-2\left\langle a^{\gamma T}\right\rangle^{2}\right\} \\
+ & 9\left\{\left\langle\left(a_{1} a_{2} a_{3}+a_{4} a_{5} a_{6}\right)^{\gamma T}\right\rangle-2\left\langle a^{\gamma T}\right\rangle^{3}\right\} \\
& +6\left\{\left\langle\left(a_{1} a_{2} a_{3}+a_{4} a_{5} a_{3}+a_{4} a_{6} a_{7}\right)^{\gamma T}\right\rangle\right. \\
& -\left[\left\langle\left(a_{1} a_{2} a_{3}+a_{4} a_{5} a_{6}\right)^{\gamma T}\right\rangle-2\left\langle a^{\gamma T}\right\rangle^{3}\right] \\
& \left.-2\left\langle a^{\gamma T}\right\rangle\left[\left\langle\left(a_{1} a_{2}+a_{3} a_{4}\right)^{\gamma T}\right\rangle-2\left\langle a^{\gamma T}\right\rangle^{2}\right]-3\left\langle a^{\gamma T}\right\rangle^{3}\right\}+\mathrm{O}\left(\gamma^{-8}\right) .
\end{aligned}
$$

In (38) the first term, of order $\gamma^{-3}$, results from cases when one of the six walks comprising $f_{3}(T)$ dominates (33c). The second and third terms (of order $\gamma^{-5}$ and $\gamma^{-6}$ ) represent cases when two of the six terms are of similar size, much larger than the rest and when these two dominant walks have one or no bonds in common, respectively. The fourth term is the effect of having three of the terms much larger than the other three, with one of the three dominant walks sharing one bond with each of the other two dominant walks. Further contributions to $\left\langle f_{3}(T)^{\gamma T}\right\rangle$ are of order $\gamma^{-8}$ or smaller.

Using the rearrangements of (37) and (38) one can rewrite $G_{3}(\gamma)$ as

$$
\begin{aligned}
G_{3}(\gamma)=\frac{1}{3 \gamma} \log [ & d^{3}\langle\exp (-\gamma \varepsilon)\rangle^{3}+d^{2}(d-1)\langle\exp (-\gamma \varepsilon)\rangle \\
& \times\left(\left\langle\left\{\exp \left[-\left(\varepsilon_{1}+\varepsilon_{2}\right) / T\right]+\exp \left[-\left(\varepsilon_{3}+\varepsilon_{4}\right) / T\right]\right\}^{\gamma T}\right\rangle-2\langle\exp (-\gamma \varepsilon)\rangle^{2}\right) \\
& +\frac{1}{2} d(d-1)(3 d-4)\left(\left\langle\left\{\exp \left[-\left(\varepsilon_{1}+\varepsilon_{2}+\varepsilon_{3}\right) / T\right]\right.\right.\right. \\
& \left.\left.\left.\left.+\exp \left[-\left(\varepsilon_{4}+\varepsilon_{5}+\varepsilon_{6}\right) / T\right]\right\}^{\gamma T}\right\rangle-2\langle\exp (-\gamma \varepsilon)\rangle^{3}\right)+\mathrm{O}\left(\gamma^{-4}\right)\right]
\end{aligned}
$$

\subsection{The n-tree approximation for large $d$}

Comparing (36) and (39), for large dimension, each of the terms in the argument of the logarithm on the RHS of the equations can be identified with a diagram in figure 4 , and this allows us to obtain an expression for $G_{n}(\gamma)$ valid for arbitrary $n$. The first term in (36) and (39) represents a single walk of two and three steps respectively and can be identified with figure $4(a)$. Its contribution is a product of the embedding count $A(a)$ times the energy factor $B(a)$ given in table 1 . The next term in (36) and (39) corresponds to figure $4(b)$, in which two walks bifurcate and rejoin immediately afterwards. The contribution is again the product $A(b) B(b)$ of table 1 . Notice that an extra factor of two appears when $n=3$ as the bifurcation can occur at the first or second step. These two diagrams constitute all the possible arrangements of walks

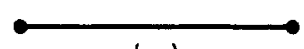

(a)

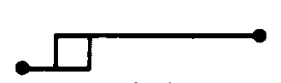

(b)

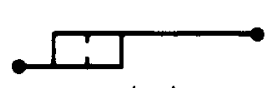

(c)

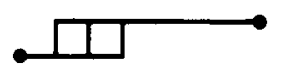

(d)

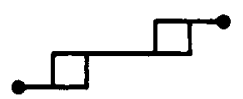

(e)

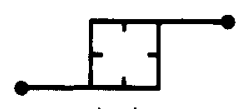

(f)

Figure 4. The first six diagrams connecting points $n$ steps apart (for $n \geqslant 4$ ). 
Table 1. The embedding counts, $A$, and energy factors, $B$, of the diagrams of figure 4 for the diagrammatic expansion.

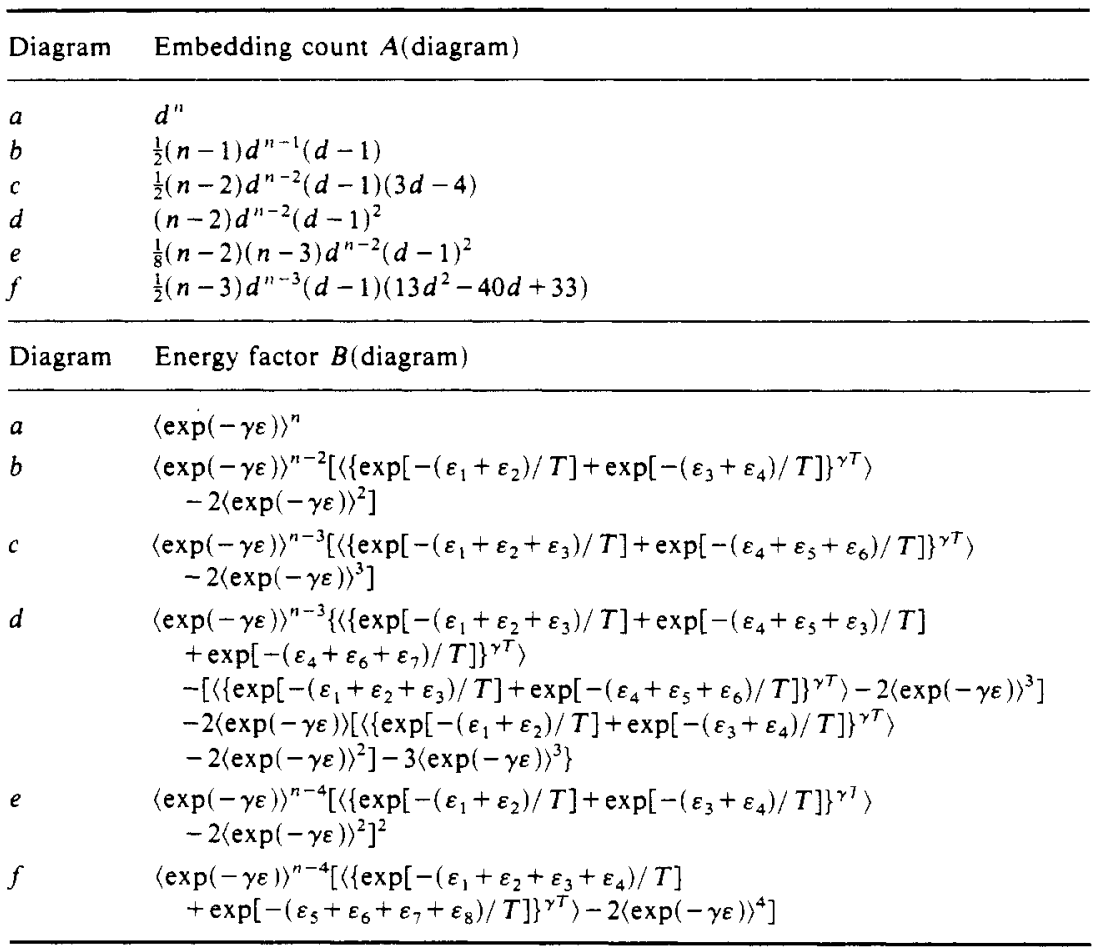

linking two points two steps apart. The third term in (39) can be identified with figure $4(c)$ in which two walks split and rejoin three steps later. Again its contribution is given by $A(c) B(c)$ in table 1 .

Hence, to be able to evaluate $G_{n}(\gamma)$ one need only consider all the different ways of linking two points $n$ steps apart, starting by utilising as few bonds as possible and gradually increasing the number of bonds used by the walks to obtain higher orders in the expansion. For each diagram one has to determine the number of ways of embedding it in the lattice, $A$ (diagram), and the corresponding energy factor, $B$ (diagram). The diagrams of figure 4 have previously been used to calculate the high-dimension expansion of the directed bond percolation threshold (Blease 1977).

Using table $1, G_{n}(\gamma)$ can be written as

$$
\begin{aligned}
G_{n}(\gamma)=\frac{1}{n \gamma} \log [ & d^{n}\langle\exp (-\gamma \varepsilon)\rangle^{n} \\
& +\frac{1}{2}(n-1) d^{n-1}(d-1)\langle\exp (-\gamma \varepsilon)\rangle^{n-2}\left(\left\langle\exp \left[-\left(\varepsilon_{1}+\varepsilon_{2}\right) / T\right]\right.\right. \\
& \left.\left.\left.+\exp \left[-\left(\varepsilon_{3}+\varepsilon_{4}\right) / T\right]\right\}^{\gamma T}\right\rangle-2\langle\exp (-\gamma \varepsilon)\rangle^{2}\right) \\
& +\frac{1}{2}(n-2) d^{n-2}(d-1)(3 d-4)(\exp (-\gamma \varepsilon)\rangle^{n-3} \\
& \times\left(\left\langle\left\{\exp \left[-\left(\varepsilon_{1}+\varepsilon_{2}+\varepsilon_{3}\right) / T\right]-\exp \left[-\left(\varepsilon_{4}+\varepsilon_{5}+\varepsilon_{6}\right) / T\right]\right\}^{\gamma T}\right\rangle\right. \\
& \left.\left.-2\langle\exp (-\gamma \varepsilon)\rangle^{3}\right)+\mathrm{O}\left(\gamma^{-4}\right)\right] \\
= & \frac{1}{n \gamma} \log \left(\sum_{\text {diagrams }} A(\text { diagram }) B(\text { diagram })\right) .
\end{aligned}
$$


In general a diagram will contribute at order $\gamma^{-p}$ in the argument of the log in (40) if this diagram contains $p$ extra bonds compared with figure $4(a)$. So to expand (40) to a given order in $1 / \gamma=\mathrm{O}(1 / d)$, one needs to consider a finite number of diagrams. Having obtained $G_{n}(\gamma)$, the free energy per unit length then follows as before (e.g. (13)-(16))

$$
F_{n}(T)= \begin{cases}-G_{n}(1 / T) & T \geqslant T_{\mathrm{c}} \\ -G_{n}\left(\gamma_{\text {min }}\right) & T \leqslant T_{\mathrm{c}}\end{cases}
$$

with

$$
\left.\frac{\mathrm{d} G_{n}(\gamma)}{\mathrm{d} \gamma}\right|_{\gamma_{\mathrm{m} 1}}=0
$$

and $T_{\mathrm{c}}$ given by (16).

Using the expansion for $G_{n}(\gamma)$ computed from table 1 (equation (40)) with the energy distribution (21), one can show that in the low-temperature phase

$$
F_{n}(T)=\frac{1}{e d}+\frac{1}{e^{2} d^{2}}+\frac{3}{2 e^{3} d^{3}}-\frac{1}{e^{3} d^{3}}\left(\frac{n-1}{n}\right)[g(\text { Ted })-3]+\mathrm{O}\left(d^{-4}\right)
$$

with

$$
g(t)=t \int_{0}^{\infty} \mathrm{d} u(2+t u)\left[\left(1+\mathrm{e}^{-u}\right)^{t}-1\right]
$$

and in the high-temperature phase (to all orders in $1 / d$ )

$$
F_{n}(T)=T \log \left(\frac{T+1}{T d}\right) \quad T \geqslant T_{\mathrm{c}} .
$$

Notice $(27 a),(42 a)$ that the first correction to the tree calculation for $T \leqslant T_{\mathrm{c}}$ occurs at order $d^{-3}$, not $d^{-2}$, because the first corrective diagram includes two extra bonds. Also, one sees from (43) that in the high-temperature phase the quenched and annealed free energies are equal (Imbrie and Spencer 1988, Cook and Derrida 1989b). From $(42 a)$ the specific heat per unit length, $C_{n}(T)$, in the low-temperature phase can be shown to be

$$
\begin{aligned}
C_{n}(T) & =\frac{T}{e d}\left(\frac{n-1}{n}\right) g^{\prime \prime}(\text { Ted })+\mathrm{O}\left(\frac{1}{\gamma^{2}}\right) \\
& \simeq \frac{T}{e d}\left(\frac{n-1}{n}\right) \frac{\pi^{2}}{3} \quad \text { for } T \text { small. }
\end{aligned}
$$

The low-temperature phase is no longer frozen as it was in the mean-field limit (Derrida and Spohn 1988). Instead one finds here a specific heat linear in temperature at low temperature. The transition temperature, $T_{c}$, can be calculated using $(41 \mathrm{c})$, (40) and (16) to yield

$$
T_{\mathrm{c}}=\frac{1}{e d}+\frac{2}{e^{2} d^{2}}+\frac{1}{e^{3} d^{3}}\left[\frac{9}{2}+\left(\frac{n-1}{n}\right) g^{\prime}(1)\right]+\mathrm{O}\left(d^{-4}\right)
$$

where $g^{\prime}(1)=7.527827 \ldots($ see $(42 b))$. 
These results (42)-(45) were obtained using figures $4(a)$ and $4(b)$. One can go to higher orders in $1 / d$ by including the next diagrams. For example, if one includes all the diagrams of figure 4 , one can obtain for the ground-state energy per unit length

$$
\begin{gathered}
E_{\mathrm{GS}}=\frac{1}{d e}+\frac{1}{(d e)^{2}}+\frac{1}{(d e)^{3}}\left(\frac{9}{2}-\frac{3}{n}\right)+\frac{1}{(d e)^{4}}\left(\frac{110}{3}-3 e+\frac{1}{n}(3 e-64)\right) \\
+\frac{1}{(d e)^{5}}\left(\frac{11297}{24}-74 e+\frac{1}{n}(144 e-1369)+\frac{1}{n^{2}} \frac{81}{2}\right)+\mathrm{O}\left(\frac{1}{d^{6}}\right) .
\end{gathered}
$$

(Notice that (46) is only valid for $n \geqslant 3$ because the embedding counts of table 1 are only valid for $n \geqslant 3$.)

The calculation of the ground-state energy in powers of $1 / d$ is technically simpler than that of the free energy because all the energy factors $B$ of table 1 can be calculated explicitly in the limit $T \rightarrow 0$ (table 2) for the exponential distribution (21).

Table 2. The energy factors, $B$, of table 1 calculated at $T=0$ for the exponential distribution (21).

\begin{tabular}{ll}
\hline Diagram & Energy factor $B($ diagram) at $T=0$ \\
\hline$a$ & $\frac{1}{(\gamma+1)^{n}}$ \\
$b$ & $\frac{-(6 \gamma+8)}{(\gamma+1)^{n}(\gamma+2)^{3}}$ \\
$c$ & $\frac{-\left(20 \gamma^{2}+50 \gamma+32\right)}{(\gamma+1)^{n}(\gamma+2)^{5}}$ \\
$d$ & $\frac{71 \gamma^{4}+555 \gamma^{3}+1581 \gamma^{2}+1937 \gamma+864}{(\gamma+1)^{n}(\gamma+2)^{5}(\gamma+3)^{3}}$ \\
$e$ & $\frac{(6 \gamma+8)^{2}}{(\gamma+1)^{\prime \prime}(\gamma+2)^{6}}$ \\
$f$ & $\frac{-\left(70 \gamma^{3}+252 \gamma^{2}+308 \gamma+128\right)}{(\gamma+1)^{n}(\gamma+2)^{7}}$ \\
\hline
\end{tabular}

All the results (41)-(46) obtained so far are $1 / d$ expansions valid for the $n$-tree problems. As the $n$-tree problem is identical to the hypercubic lattice in the limit $n \rightarrow \infty$, we expect these results $(41)-(46)$ to be valid for the hypercubic lattice in the limit $n \rightarrow \infty$.

So the $1 / d$ expansion of the free energy, the specific heat and the ground-state energy for the hypercubic lattice are given by putting $n=\infty$ in (42), (44) and (46).

For these results to be correct, one needs the limits $n \rightarrow \infty$ and $d \rightarrow \infty$ to commute. We have not been able to prove that these two limits commute. However, in all the $1 / d$ expansions presented in this paper, the coefficients are always simple rational functions of $n$ which have a finite limit for $n \rightarrow \infty$. So there is no indication that the limit $n \rightarrow \infty$ is singular for large $d$. Moreover, we shall see in section 7 that there are some cases which can be solved without the $n$-tree approximation and for which the exchange of these limits $n \rightarrow \infty$ and $d \rightarrow \infty$ gives the right answer. 


\section{The fluctuations of the transverse displacement}

In this section we calculate the fluctuations of the transverse displacement of the polymer. We shall see that one can define two types of transverse fluctuations: thermal and disorder fluctuations. Then the $1 / d$ expansion, presented in the previous section, will be extended to include a chemical potential, enabling one to calculate the transverse fluctuations on a $d$-dimensional hypercubic lattice.

Let us first define what is meant by transverse fluctuations. Consider the problem of polymers on a $d$-dimensional hypercubic lattice, directed along the $(1,1, \ldots, 1)$ direction, as described in section 3. If a polymer of length $L$, emanating from the origin, ends at the point $\left(x_{1}, \ldots, x_{d}\right)$, its transverse displacement is given by the vector $\boldsymbol{R}$

$$
\boldsymbol{R}=\left(x_{1}-\frac{L}{d}, \ldots, x_{d}-\frac{L}{d}\right)=\left(R_{1}, \ldots, R_{d}\right) .
$$

We want to consider the fluctuations in the quantities $R_{i}(i=1, \ldots, d)$. These can be of two types: thermal fluctuations, given by

$$
\left\langle\overline{R_{i}^{2}}-\overline{\boldsymbol{R}}_{i}^{2}\right\rangle
$$

and disorder fluctuations, given by

$$
\left\langle\bar{R}_{i}^{2}\right\rangle-\left\langle\bar{R}_{i}\right\rangle^{2}=\left\langle\bar{R}_{i}^{2}\right\rangle
$$

since $\left\langle\bar{R}_{i}\right\rangle=0$ for symmetry reasons.

We use the notation that \langle\rangle denotes an average over disorder and ${ }^{-}$a configurational or thermal average. Often the quantity $\sum_{i=1}^{d}\left\langle\bar{R}_{i}^{2}\right\rangle$ is considered; this scales as the length of the polymer, $L$, to an exponent $2 \nu$, i.e.

$$
\sum_{i=1}^{d}\left\langle\bar{R}_{i}^{2}\right\rangle=d\left\langle\bar{R}_{i}^{2}\right\rangle \sim L^{2 *}
$$

As was mentioned in section 1 , little is known analytically about $\nu$ as a function of dimension except that $\nu=\frac{1}{2}$ in the mean-field limit and that $\nu=\frac{2}{3}$ in $d=1+1$ dimensions.

\subsection{The relation between thermal and disorder fluctuations}

Let us now derive a relation between thermal and disorder fluctuations valid for any tree problem. Although the notion of transverse displacement has no a priori meaning in a tree problem, we can identify each direction $1 \leqslant i \leqslant d$ of a tree of $d$ branches with one direction $i$ on the hypercubic lattice and say that a walk on a tree has a transverse displacement $R_{i}$ if it does $L / d+R_{i}$ steps along the $i$ th branch.

Let us now attempt to evaluate the fluctuations (48) for the tree problem in the low-temperature phase. We have seen, (19) and (20), that in the low-temperature phase the overlaps are either 0 or 1 . This implies that there are well defined valleys and that the thermal fluctuations inside a valley vanish. Therefore one can think of a landscape composed of a number of valleys. Each valley $\alpha$ is characterised by some transverse displacement $R_{1}=c_{\alpha}$ in direction 1 and by a weight $\omega_{\alpha}$. Because the overlap between two walks inside the same valley is 1 , all the walks inside the same valley $\alpha$ have the same transverse displacement $c_{\alpha}$. Moreover, since the overlap between two different valleys is 0 , the displacements $c_{\alpha}$ and $c_{\beta}$ for two valleys are uncorrelated. Therefore one expects that the $c_{\alpha}$ satisfy

$$
\left\langle c_{\alpha}\right\rangle=0 \quad\left\langle c_{\alpha} c_{\beta}\right\rangle=\left\langle c_{\alpha}\right\rangle\left\langle c_{\beta}\right\rangle \quad \text { if } \alpha \neq \beta
$$


where \langle\rangle is the disorder average. Since the only thermal fluctuations are due to relative weights, $\omega_{\alpha}$, of the valleys, one has

$$
\begin{aligned}
& \overline{R_{1}}=\sum_{\alpha} c_{\alpha} \omega_{\alpha} \\
& \overline{R_{1}^{2}}=\sum_{\alpha} c_{\alpha}^{2} \omega_{\alpha} .
\end{aligned}
$$

To obtain the thermal and the disorder fluctuations, we now need to average over disorder. The weights $\omega_{\alpha}$ satisfy

$$
\begin{aligned}
& \sum_{\alpha} \omega_{\alpha}=1 \\
& \left\langle\sum_{\alpha} \omega_{\alpha}^{2}\right\rangle=1-T \gamma_{\min } .
\end{aligned}
$$

Equation ( $52 a$ ) expresses the fact that the weights of the valleys must add up to unity and $(52 b)$ is derived in appendix 1 (equation (A1.16)). If we consider that the $c_{\alpha}$ and the $\omega_{\alpha}$ are not correlated, we get

$$
\begin{aligned}
& \left\langle\overline{R_{1}}\right\rangle=0 \\
& \left\langle\overline{R_{1}^{2}}\right\rangle=\left\langle\sum_{\alpha} \omega_{\alpha}\right\rangle\left\langle c_{\alpha}^{2}\right\rangle=\left\langle c_{\alpha}^{2}\right\rangle \\
& \left\langle\bar{R}_{1}^{2}\right\rangle=\left\langle\sum_{\alpha} \omega_{\alpha}^{2}\right\rangle\left\langle c_{\alpha}^{2}\right\rangle=\left(1-T \gamma_{\min }\right)\left\langle c_{\alpha}^{2}\right\rangle
\end{aligned}
$$

which give for the thermal fluctuations

$$
\left\langle\overline{R_{1}^{2}}\right\rangle-\left\langle\bar{R}_{1}^{2}\right\rangle=\left\langle c_{\alpha}^{2}\right\rangle T \gamma_{\min } \quad T \leqslant T_{c}
$$

and for the disorder fluctuations:

$$
\left\langle\bar{R}_{1}^{2}\right\rangle=\left\langle c_{\alpha}^{2}\right\rangle\left(1-T \gamma_{\min }\right) \quad T \leqslant T_{\mathrm{c}} .
$$

The disorder and thermal fluctuations take on similar forms, their ratio being (1$\left.T \gamma_{\min }\right) / T \gamma_{\min }$. The disorder fluctuations vanish at $T_{c}$. This result is very similar to the fact that at $T_{c}$ the quenched and annealed free energies become identical.

\subsection{Calculation of the thermal fluctuations}

We shall now study the transverse fluctuations on the hypercubic lattice using the $1 / d$ expansion. This can be done by adding a chemical potential, $\mu$, to count the number of steps in direction 1

$$
Z_{L}(\boldsymbol{r})=\sum_{w} \exp \left[-E_{w} / T+\mu\left(R_{1}(w)+L / d\right)\right]
$$

where the sum runs over all walks of length $L$ emanating from the point $r$ and $\left(R_{1}(w)+L / d\right)$ is the number of steps of walk $w$ in direction 1 .

The average number of steps taken in the direction marked by $\mu$, direction 1 , is then given by

$$
\left\langle\bar{R}_{1}\right\rangle+\frac{L}{d}=\left.\frac{\mathrm{d}}{\mathrm{d} \mu}\left\langle\log Z_{L}\right\rangle\right|_{\mu=0}
$$

and the thermal fluctuation of $R_{1}$ is given by the second derivative of $\left(\log Z_{\mathrm{L}}\right\rangle$

$$
\left\langle\overline{R_{1}^{2}}\right\rangle-\left\langle\bar{R}_{1}^{2}\right\rangle=\left.\frac{\mathrm{d}^{2}}{\mathrm{~d} \mu^{2}}\left\langle\log Z_{L}\right\rangle\right|_{\mu=0} .
$$


The method of $n$-tree approximations and the resulting $1 / d$ expansion can be used as in section 3 to calculate the free energy of problem (56), providing that care is taken to include an extra factor, $\mathrm{e}^{\mu \gamma T}$, for each step taken in the marked direction. For example, the free energy per unit length of the 1-tree approximation, $F_{1}(T, \mu)$, can be obtained using (41) from $G_{1}(\gamma)$ :

$$
G_{1}(\gamma)=\frac{1}{\gamma} \log \left[\left(\frac{d-1+\mathrm{e}^{\mu \gamma T}}{\gamma+1}\right)\right]
$$

where the distribution ( 21$)$ has again been used. The inclusion of the chemical potential has changed the embedding count of figure $4(a)$ from $d^{n}$ to $\left(d-1+\mathrm{e}^{\mu \gamma T}\right)^{n}$. The embedding counts for the diagrams of figure 4, obtained using the chemical potential, are given in table 3 . Combining the new embedding counts (table 3 ) with the energy factors (table 1), as in (40), it is then possible to find $G_{n}(\gamma)$ and hence the $1 / d$ expansion for the free energy, $F_{n}(T, \mu)$.

From $F_{n}(T, \mu)$ one can calculate the thermal fluctuations on the $n$-tree (58) by

$$
\left\langle\overline{R_{1}^{2}}\right\rangle-\left\langle\bar{R}_{1}^{2}\right\rangle=-\left.\frac{L}{T} \frac{\mathrm{d}^{2}}{\mathrm{~d}_{\mu}^{2}} F_{n}(T, \mu)\right|_{\mu=0} .
$$

This formula is valid at all temperatures.

\subsection{The disorder fluctuations in the low-temperature phase}

From (54), (55) and (60) one can obtain the disorder fluctuations.

$$
\left\langle\bar{R}_{1}^{2}\right\rangle=-\left.\frac{\left(1-T \gamma_{\min }\right)}{T^{2} \gamma_{\min }} L \frac{\mathrm{d}^{2}}{\mathrm{~d}_{\mu}^{2}} F_{n}(T, \mu)\right|_{\mu=0} .
$$

Notice that (61) is only valid for a tree problem, since (54) and (55) only apply to this case.

\begin{tabular}{|c|c|}
\hline Diagram & Embedding count \\
\hline$a$ & $\left(d-1+\mathrm{e}^{\mu \gamma T}\right)^{n}$ \\
\hline$b$ & $\frac{1}{2}(n-1)(d-1)^{n-1}\left(1+\frac{\mathrm{e}^{\mu \gamma T}}{d-1}\right)^{n-2}\left(d-2+2 \mathrm{e}^{\mu \gamma T}\right)$ \\
\hline$c$ & $\begin{array}{l}\frac{1}{2}(n-2)(d-1)^{n-2}\left(1+\frac{\mathrm{e}^{\mu \gamma T}}{d-1}\right)^{n-3}\left(3 d^{2}-13 d+14+(9 d-16) \mathrm{e}^{\mu \gamma T}\right. \\
\left.\quad+2 \mathrm{e}^{2 \mu \gamma T}\right)\end{array}$ \\
\hline$d$ & $(n-2)(d-1)^{n-2}\left(1+\frac{\mathrm{e}^{\mu \gamma T}}{d-1}\right)^{n-3}\left(d^{2}-4 d+4+(3 d-5) \mathrm{e}^{\mu \gamma T}+\mathrm{e}^{2 \mu \nu T}\right)$ \\
\hline$e$ & $\frac{1}{8}(n-2)(n-3)(d-1)^{n-2}\left(1+\frac{\mathrm{e}^{\mu \gamma T}}{d-1}\right)^{n-4}\left(d-2+2 \mathrm{e}^{\mu \gamma \tau}\right)^{2}$ \\
\hline$f$ & $\begin{array}{l}(n-3)(d-1)^{n-3}\left(1+\frac{\mathrm{e}^{\mu \gamma T}}{d-1}\right)^{n-4}\left[\frac{13}{2} d^{3}-46 d^{2}+109 d-86\right. \\
\left.+\left(26 d^{2}-105 d+107\right) \mathrm{e}^{\mu \gamma T}+\left(\frac{25}{2} d-22\right) \mathrm{e}^{2 \mu \gamma T}+\mathrm{e}^{3 \mu \gamma T}\right]\end{array}$ \\
\hline
\end{tabular}

Table 3. The embedding counts, $A$, for the diagrams of figure 4 when a chemical potential, $\mu$, is included to count the number of steps in the marked direction. 
From $(60),(61)$ one could obtain the complete temperature dependence of both the thermal and disorder fluctuations. However, as the calculations are greatly simplified at zero temperature we shall give the results in this limit only. At $T=0$ the thermal fluctuations vanish, whereas the disorder fluctuations are given by

$$
\begin{aligned}
\sum_{i=1}^{d}\left\langle\overline{R_{i}^{2}}\right\rangle=L[ & 1-\frac{1}{d}+\frac{6}{e^{2} d^{3}}\left(\frac{n-1}{n}\right)+\frac{(136-6 e) n-276+6 e}{n e^{3} d^{4}} \\
& \left.+\frac{(3384-376 e) n+756 e-10454+(108 / n)}{n e^{4} d^{5}}+\mathrm{O}\left(d^{-6}\right)\right] .
\end{aligned}
$$

Taking the limit $n \rightarrow \infty$ one gets a $1 / d$ expansion for the disorder transverse fluctuation at zero temperature for the hypercubic lattice. As for the free energy, each term of the expansion has a regular $n$-dependence when $n \rightarrow \infty$ and so it is reasonable to assume that the limits $d \rightarrow \infty$ and $n \rightarrow \infty$ can be exchanged.

We see from (62) that the $1 / d$ expansion does not change the value of $\nu$, which remains $\frac{1}{2}$. This would be consistent with the fact that $\nu=\frac{1}{2}$ above a certain upper critical dimension, $d_{c}$, at which the coefficient of $L$ (in the RHS of (62) and for $n \rightarrow \infty$ ) would diverge. The series (62) is, however, too short to make any reliable prediction for $d_{c}$. Moreover, it could happen that the $1 / d$ series (62) (for $n=\infty$ ) has a zero radius of convergence, as is the case in some 1/d expansions (Fisher and Singh 1989).

The transverse fluctuations have an interesting temperature dependence. Let us just discuss now their behaviour to leading order in $1 / d$. From (59), (60) and (61), one finds that the thermal fluctuations are given by,

$$
\sum_{i=1}^{d}\left\langle\overline{R_{i}^{2}}\right\rangle-\left\langle\bar{R}_{i}^{2}\right\rangle= \begin{cases}L\left(1-\frac{1}{d}\right) \frac{T}{T_{\mathrm{c}}}+\mathrm{O}\left(\frac{1}{d^{2}}\right) & \text { for } T \leqslant T_{\mathrm{c}} \\ L\left(1-\frac{1}{d}\right) & \text { for } T>T_{\mathrm{c}}\end{cases}
$$

and the disorder fluctuations are given by

$$
\sum_{i=1}^{d}\left\langle\bar{R}_{i}^{2}\right\rangle= \begin{cases}L\left(1-\frac{1}{d}\right)\left(1-\frac{T}{T_{\mathrm{c}}}\right)+\mathrm{O}\left(\frac{1}{d^{2}}\right) & \text { for } T \leqslant T_{\mathrm{c}} \\ 0 & \text { for } T>T_{\mathrm{c}}\end{cases}
$$

(see figure 5).

The fact that we have two kinds of transverse fluctuations in this problem is reminiscent of the susceptibility of spin glasses for which, at low temperature, two susceptibilities can be defined (DC susceptibility and AC susceptibility, in the limit of zero frequency).

The inclusion of a chemical potential, as in (56), also enables one to consider the problem of polymers in a random medium in different geometries. For example, one can consider the problem in which the partition sum (2) runs over all walks with a fixed number of steps, $N$, in a given direction rather than over all walks of fixed length $L$. This problem is related to a problem considered by Dhar (1988), who showed that, on a $d$-dimensional hypercubic lattice, the ground-state energy for distribution (21) is given to leading order by $\log d / d$, for $d$ large. Using our $1 / d$ expansion method based on the $n$-tree approximation, it is possible to recover this leading mean-field term and calculate corrections to it. 


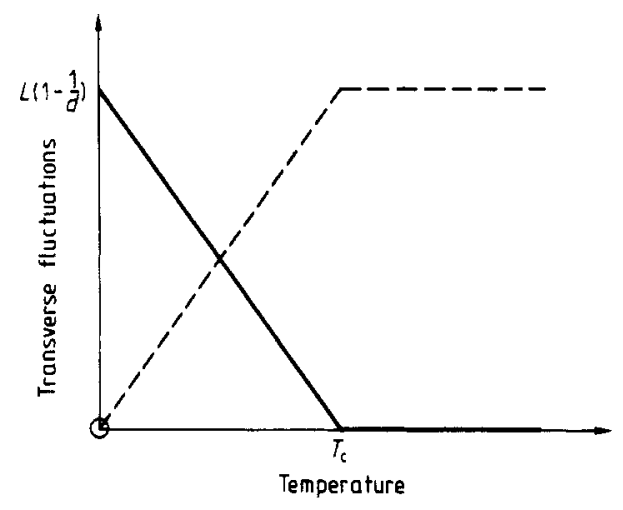

Figure 5. The temperature dependence of disorder (full line) and thermal (broken line) transverse fluctuation, equations (63), (64).

\section{The overlaps}

As mentioned in section 1 , the problem of directed polymers in random media has a close analogy with spin glasses. The mean-field spin glass (Parisi 1980a, b) and the mean-field polymer problem (Derrida and Spohn 1988) both have low-temperature phases characterised by broken replica symmetry. This is displayed in the polymer problem by the probability distribution of overlaps, $P_{2}(q)$ (see (17) and (18) for definition), being a sum of two delta functions (see appendix 1, equation (A1.14) and section 2). The question of replica symmetry breaking in the finite-dimensional spin glass remains controversial and for this reason it is interesting to examine the overlaps for the polymer problem in finite dimension. In this section, therefore, we shall study the overlaps on a $d$-dimensional hypercubic lattice by extending the $n$-tree approach, obtaining the probability distribution of the overlap of two walks (70), (71).

The $n$-tree approximations to the directed polymer problem, defined in section 3 , retain a general tree structure. Hence, if one performs the replica calculation of appendix 1 on the $n$-tree one will see that, below $T_{c}, P_{2}(q)$ remains a sum of two delta functions with weights $T \gamma_{\min }$ and $1-T \gamma_{\min }$. However, in the $n$-tree approximation some of the branches of the tree structure are composed of two or more paths. Consider what can occur when two walks pass down such a branch. The walks can both take the same path, giving an overlap of 1 for this section, or they can take different routes, giving an overlap less than 1 for this section. So, instead of having one delta function at $q_{2}=0$ and the other at $q_{2}=1$, the latter will be shifted to a lower value of $q_{2}$.

To explain how one can calculate the position of the shifted delta function, let us consider as an example a polymer of length $L$ in the 2-tree approximation. Here one has only two kinds of branch (see figure $2(b)$ ): those which consist of only one path (type A), and so contribute $2 / L$ to the overlap $q_{2}$ and those which are made up of two paths (type B), which contribute between $1 / L$ (at $T=\infty$ ) and $2 / L$ (at $T=0$ ) to $q_{2}$, depending on the temperature. To determine the distribution function of the overlaps, one therefore needs to know the fraction of each type of branch used by the walks and the contribution to the overlap from the type B branches, both as functions of temperature.

As is shown in appendix 2, the typical fraction of branches used in the walk that are of type $B$ and have energies $\varepsilon_{1}+\varepsilon_{2}$ and $\varepsilon_{3}+\varepsilon_{4}$ on the constituent links is given, in 
the low-temperature phase, by

$$
\begin{aligned}
& \frac{1}{2} d(d-1)\left\{\exp \left[-\left(\varepsilon_{1}+\varepsilon_{2}\right) / T\right]+\exp \left[-\left(\varepsilon_{3}+\varepsilon_{4}\right) / T\right]\right\}^{T \gamma_{m ! n}} \rho\left(\varepsilon_{1}\right) \rho\left(\varepsilon_{2}\right) \rho\left(\varepsilon_{3}\right) \rho\left(\varepsilon_{4}\right) \\
& \times \exp \left[-2 \gamma_{\min } G_{2}\left(\gamma_{\min }\right)\right]
\end{aligned}
$$

where $G_{2}(\gamma)$ is given by $(36)$.

It now remains to calculate the contribution to the overlap, $q_{m}$, of such a branch. This is simply given by

$$
\frac{\exp \left[-m\left(\varepsilon_{1}+\varepsilon_{2}\right) / T\right]+\exp \left[-m\left(\varepsilon_{3}+\varepsilon_{4}\right) / T\right]}{\left\{\exp \left[-\left(\varepsilon_{1}+\varepsilon_{2}\right) / T\right]+\exp \left[-\left(\varepsilon_{3}+\varepsilon_{4}\right) / T\right]\right\}^{m}}
$$

as one has an overlap 1 if all the walks go through the same path and overlap 0 otherwise. Combining (65) and (66), one can conclude that $P_{2}(q)$ for the 2-tree consists of two delta functions, one at $q=0$ with weight $T \gamma_{\min }$ and the other at

$$
\begin{aligned}
q_{2}=(d\langle\exp (- & \left.\left.\varepsilon \gamma_{\min }\right)\right\rangle^{2}+\frac{1}{2} d(d-1)\left\langle\left\{\exp \left[-\left(\varepsilon_{1}+\varepsilon_{2}\right) / T\right]+\exp \left[-\left(\varepsilon_{3}+\varepsilon_{4}\right) / T\right]\right\}^{T \gamma_{\min }-2}\right. \\
& \left.\left.\times\left\{\exp \left[-2\left(\varepsilon_{1}+\varepsilon_{2}\right) / T\right]+\exp \left[-2\left(\varepsilon_{3}+\varepsilon_{4}\right) / T\right]\right\}\right\rangle\right) \\
& \times \exp \left[-2 \gamma_{\min } G_{2}\left(\gamma_{\min }\right)\right] .
\end{aligned}
$$

Having seen how the calculation proceeds for the 2-tree, it is easy to generalise to the $n$-tree. The relative contribution to $q_{m}$ from each branch like figure $4(a)$ is always 1 . The relative contribution from branches like figure $4(b)$ will be

$$
1-\frac{2}{n}+\frac{2}{n} \frac{\exp \left[-m\left(\varepsilon_{1}+\varepsilon_{2}\right) / T\right]+\exp \left[-m\left(\varepsilon_{3}+\varepsilon_{4}\right) / T\right]}{\left\{\exp \left[-\left(\varepsilon_{1}+\varepsilon_{2}\right) / T\right]+\exp \left[-\left(\varepsilon_{3}+\varepsilon_{4}\right) / T\right]\right\}^{m}}
$$

This is just the direct generalisation of $(66)$ : the walks have to be together for a fraction $1-2 / n$ of the branch and can then take two paths for a fraction $2 / n$ of the branch. So for the $n$-tree the average overlap $\left\langle q_{2}\right\rangle$ takes the form

$$
\begin{aligned}
&\left\langle q_{2}\right\rangle=\left[1-\left(\frac{n-1}{n}\right) \frac{(d-1)}{d}\left\langle\exp \left(-\varepsilon \gamma_{\min }\right)\right\rangle^{-2}\right. \\
& \times\left(\left\langle\left\{\exp \left[-\left(\varepsilon_{1}+\varepsilon_{2}\right) / T\right]+\exp \left[-\left(\varepsilon_{3}+\varepsilon_{4}\right) / T\right]\right\}^{\left.T \gamma_{\min }\right\rangle}\right.\right. \\
&-\left\langle\left\{\exp \left[-\left(\varepsilon_{1}+\varepsilon_{2}\right) / T\right]+\exp \left[-\left(\varepsilon_{3}+\varepsilon_{4}\right) / T\right]\right\}^{T \gamma_{\min }-2}\right. \\
&\left.\left.\times\left\{\exp \left[-2\left(\varepsilon_{1}+\varepsilon_{2}\right) / T\right]+\exp \left[-2\left(\varepsilon_{3}+\varepsilon_{4}\right) / T\right]\right\}\right\rangle\right) \\
&\left.+\mathrm{O}\left(d^{-3}\right)\right]\left(1-T \gamma_{\min }\right)
\end{aligned}
$$

where $\gamma_{\min }$ is given by (40) and (41).

Evaluating the averages, using (21) and taking the limit $n \rightarrow \infty$ (again assuming the commutativity of limits) yields that the delta function at non-zero overlap in $P_{2}(q)$ is shifted from $q_{2}=1$ to

$$
q_{2}=1-\frac{4 T e d}{(e d)^{2}} \int_{0}^{x} \mathrm{~d} u(2+T e d u)\left(1+\mathrm{e}^{-u}\right)^{T e d} \frac{\mathrm{e}^{-u}}{\left(1+\mathrm{e}^{-u}\right)^{2}} .
$$

The distribution function $P_{2}(q)$ remains non-trivial in finite dimension in the low-temperature phase

$$
P_{2}(q)=T \gamma_{\min } \delta(q)+\left(1-T \gamma_{\min }\right) \delta\left(q-q_{2}\right)
$$


where $q_{2}$ is given by $(70)$ and $\gamma_{\min }$ is

$$
\gamma_{\min }=e d-2+\frac{1}{e d}\left[-\frac{19}{2}+3 g(T e d)-T e d g^{\prime}(T e d)\right]+\mathrm{O}\left(d^{-2}\right)
$$

and where $g(t)$ is given by $(42 b)$.

So our $1 / d$ expansion predicts a broken replica symmetry in the low-temperature phase in finite dimension.

One could extend the calculations of this section to overlaps between more than two walks and one would obtain expressions very similar to (70).

\section{Generalisation to other distributions}

In this section we discuss briefly the effect of using energy distributions other than $(21)$. In sections 3, 4 and 5 the high-dimension expansions were derived using the energy distribution $(21), \rho(\varepsilon)=\exp (-\varepsilon)$. This choice facilitated the calculation of the averages in table 1 (see table 2). It is possible to perform the same calculations using other distributions, $\rho(\varepsilon)$, for the disorder. However, for most choices, $\rho(\varepsilon)$, it is not possible to express the mean-field results (26)-(28) in a simple way in powers of $d$, and it is difficult to calculate the energy factors required for the diagrammatic expansion (table 1). To examine the effect of using different energy distributions, we shall therefore limit ourselves to

$$
\rho(\varepsilon)=\frac{\varepsilon^{\alpha-1} \exp (-\varepsilon)}{\Gamma(\alpha)} \quad \alpha>0, \varepsilon \geqslant 0 .
$$

This choice allows one to study changes produced by varying the parameter $\alpha$, whilst still keeping the calculations relatively simple.

Let us first consider how the value of $\alpha$ affects the mean-field results. Using $\rho(\varepsilon)$ given by (73) with (24)-(26), one can show that, for large $d$, the mean-field energy in the low-temperature phase and transition temperature are given by

$$
F_{1}(T)=\frac{\alpha}{e d^{1 / \alpha}}+\frac{\alpha}{\left(e d^{1 / \alpha}\right)^{2}}+O\left(d^{-3 / \alpha}\right) \quad T \leqslant T_{c}
$$

and

$$
T_{\mathrm{c}}=\frac{1}{e d^{1 / \alpha}}+\frac{2}{\left(e d^{1 / \alpha}\right)^{2}}+\mathrm{O}\left(d^{-3 / \alpha}\right)
$$

One can see that, in general, we have an expansion in powers of $d^{1 / \alpha}$ rather than $d$. However, when one moves away from the mean field, by evaluating the energy factors of table 1 for the diagrammatic expansion, one sees that these still behave as powers of $d$, not $d^{1 / \alpha}$. For example the energy factor corresponding to figure $4(b)$ is given by

$$
\begin{gathered}
\left\langle\left(\exp \left[-\left(\varepsilon_{1}+\varepsilon_{2}\right) / T\right]+\exp \left[-\left(\varepsilon_{3}+\varepsilon_{4}\right) / T\right]\right)^{\gamma T}\right\rangle-2\langle\exp (-\gamma \varepsilon)\rangle^{2} \\
=-\frac{\Gamma(4 \alpha)}{\alpha \Gamma^{2}(2 \alpha) \gamma^{4 \alpha}}+2 \frac{g_{\alpha}(t)}{\gamma^{4 \alpha}}+O\left(\frac{1}{\gamma^{4 \alpha+1}}\right)
\end{gathered}
$$

where

$$
g_{\alpha}(t)=\frac{t}{\Gamma^{2}(2 \alpha)} \int_{0}^{\infty} \int_{0}^{\infty} \mathrm{d} x \mathrm{~d} y x^{2 \alpha-1} \mathrm{e}^{-x}(x+t y)^{2 \alpha-1}\left[\left(1+\mathrm{e}^{-y}\right)^{t}-1\right]
$$


So it still behaves as $d^{-4}$. When $\alpha=1, g_{\alpha}(t) \equiv g(t)$ defined in (42b). Hence, for general $\alpha$, one has an expansion in terms of $\gamma^{-\alpha}$ and $\gamma^{-1}$ (or $d^{-1}$ and $d^{-1 / \alpha}$ ) to deal with. This makes calculations rather complicated and so we shall limit ourselves here to considering the leading behaviour of the specific heat.

The specific heat can still be found easily for arbitrary $\alpha$. To do this in the low-temperature phase the behaviour of the function $g_{\alpha}(t)$ must be known for small $t$. This behaviour changes at $\alpha=\frac{1}{4}$. In the range $0<\alpha \leqslant \frac{1}{4}, g_{\alpha}(t)$ behaves as $t^{4 \alpha+1}$ for small $t$, whereas for $\alpha \geqslant \frac{1}{4}$ this changes to a $t^{2}$ dependence. This produces two possible behaviours for the low-temperature specific heat of the $n$-tree:

$$
C_{n}= \begin{cases}\frac{\pi^{2}}{6} \frac{\Gamma(4 \alpha-1)}{\Gamma^{2}(2 \alpha)}\left(\frac{n-1}{n}\right) \frac{T}{\left(e d^{1 / \alpha}\right)^{2 \alpha-1}} & \alpha \geqslant \frac{1}{4} \\ 4 \alpha(4 \alpha+1) \frac{J(\alpha)}{\Gamma^{2}(2 \alpha)}\left(\frac{n-1}{n}\right)\left(e d^{1 / \alpha}\right)^{2 \alpha} T^{4 \alpha} & 0<\alpha \leqslant \frac{1}{4}\end{cases}
$$

where

$$
J(\alpha)=\int_{0}^{\infty} \int_{0}^{\infty} \mathrm{d} x \mathrm{~d} y x^{4 \alpha-1}\left(y+y^{2}\right)^{2 \alpha-1} \log \left(1+\mathrm{e}^{-x}\right) .
$$

Notice that for $\alpha \geqslant \frac{1}{4}$ one obtains a linear behaviour at low temperature, whilst for $0<\alpha<\frac{1}{4}$ one has a $T^{4 \alpha}$ behaviour. The low-temperature behaviour of the specific heat clearly depends on the nature of the disorder.

One should not be surprised by this change of behaviour for small values of $\alpha$ because one expects from (73) that the density of excitations around the ground state will increase as $\alpha$ becomes small.

\section{Tests of the validity of the $n$-tree approximation method}

The basic premise of the high-dimension expansion method, which has been presented in the preceding sections, is that results valid for the $d$-dimensional hypercubic lattice can be obtained by expanding the $n$-tree approximation for high dimension and then taking the limit $n \rightarrow \infty$. As was discussed in the closing paragraph of section 3 , this procedure entails exchanging the limits $n \rightarrow \infty$ and $d \rightarrow \infty$. Although we have found that all quantities calculated so far have a finite limit for $n \rightarrow \infty$ when one uses this method, we have, as yet, no proof that these limits commute. In this section, therefore, we shall present a few problems for which the results can be tested (either against a solution obtained by a different approach, or against the results of simulations). First, we shall discuss the calculation, in high dimension, of the directed percolation threshold, $p_{\mathrm{c}}$. Then we shall compare the results of the $n$-tree approximation with those of numerical simulation in dimension $d=2$. Lastly we shall introduce the problem of directed polymers on a simplex and show that the $n$-tree approximation leads to the right answer for this problem.

\subsection{The percolation threshold for $d$ large}

The first problem we shall consider, that of directed bond percolation, is simply the directed polymer problem with the random energies, $\varepsilon_{i j}$, chosen according to the distribution

$$
\rho(\varepsilon)=p \delta(\varepsilon)+(1-p) \delta(\varepsilon-1)
$$


with $0 \leqslant p \leqslant 1$. Again we shall look at the problem on a $d$-dimensional hypercubic lattice, directing the walks, which emanate from the origin, along the $(1,1, \ldots, 1)$ direction. If the weight $p$ exceeds the percolation threshold, $p_{c}$, the ground-state energy per unit length is zero. The aim is to calculate this threshold, $p_{c}$, as a function of dimension, for $d$ large.

Using the diagrammatic expansion method of section 3, one sees, from (40) and (41), that the ground-state energy per unit length, $E_{\mathrm{GS}}$, of the $n$-tree approximation is given by

$$
E_{\mathrm{GS}}=-\left.G_{n}\left(\gamma_{\min }\right)\right|_{T=0}
$$

where

$$
\begin{aligned}
\left.G_{n}(\gamma)\right|_{T=0}= & \frac{1}{n \gamma} \log \left[d^{n}\langle\exp (-\gamma \varepsilon)\rangle^{n}+\frac{1}{2}(n-1) d^{n-1}(d-1)\langle\exp (-\gamma \varepsilon)\rangle^{n-2}\right. \\
& \left.\quad \times\left(\left\langle\max \left(\exp \left[-\left(\varepsilon_{1}+\varepsilon_{2}\right) \gamma\right], \exp \left[-\left(\varepsilon_{3}+\varepsilon_{4}\right) \gamma\right]\right)\right\rangle-2(\exp (-\gamma \varepsilon)\rangle^{2}\right)+\ldots\right]
\end{aligned}
$$

and $\gamma_{\min }$ is defined as usual by $(41 c)$.

Using the percolation distribution (78), the averages can be performed to yield

$$
\begin{aligned}
\left.G_{n}(\gamma)\right|_{T=0}= & \frac{1}{n \gamma} \\
& \log \llbracket d^{n}\left[p+(1-p) \mathrm{e}^{-\gamma}\right]^{n}+\frac{1}{2}(n-1) d^{n-1}(d-1)\left[p+(1-p) \mathrm{e}^{-\gamma}\right]^{n-2} \\
& \times\left\{2 p^{2}-p^{4}+4 p(1-p)^{2} \mathrm{e}^{-\gamma}+(1-p)^{4} \mathrm{e}^{-2 \gamma}\right. \\
& \left.\left.-2\left[p+(1-p) \mathrm{e}^{-\gamma}\right]^{2}\right\}+\ldots\right] .
\end{aligned}
$$

To obtain the percolation threshold, $p_{\mathrm{c}}$, we require that when $p \geqslant p_{\mathrm{c}}$ the ground-state energy is zero. So when $p \geqslant p_{\mathrm{c}}$ the value, $\gamma_{\min }$, of $\gamma$ that minimises $G_{n}(\gamma)$ is $\gamma_{\min }=\infty$. It is possible to show that $\gamma_{\min }=\infty$ as long as the argument of the logarithm in (80) is larger than 1 for $\gamma=\infty$. Therefore the percolation threshold is given by the condition that the argument of the logarithm is 1 for $\gamma=\infty$. This implies that

$$
d^{n} p_{\mathrm{c}}^{n}\left[1-\left(\frac{n-1}{2}\right)\left(1-\frac{1}{d}\right) p_{\mathrm{c}}^{2}+\mathrm{O}\left(p_{\mathrm{c}}^{3}\right)\right]=1
$$

which becomes, when one includes higher-order diagrams,

$$
\sum_{\text {diagrams }} A(\text { diagram }) B(\text { diagram })=1
$$

where the embedding counts, $A$, are the same as in table 1 and the factors $B$ are now given for the percolation distribution (78) by table 4 . As one needs here only $G_{n}(\gamma=\infty)$

Table 4. Energy factors, $B$, for the diagrams of figure 4 for the percolation distribution $\rho(\varepsilon)=p \delta(\varepsilon)+(1-p) \delta(\varepsilon-1)$ in the limit $\gamma=\infty$.

\begin{tabular}{ll}
\hline Diagram & Percolation factor \\
\hline$a$ & $p^{\prime \prime}$ \\
$b$ & $-p^{n+2}$ \\
$c$ & $-p^{n+3}$ \\
$d$ & $p^{n+4}$ \\
$e$ & $p^{n+4}$ \\
$f$ & $-p^{n+4}$ \\
\hline
\end{tabular}


for the distribution (78), the energy factors $B$ depend only on $p$ and are related to the percolation probability of each diagram. The solution, $p_{c}$, of $(81)$ and (82) can be expanded in $1 / d$, one gets

$$
p_{c}=\frac{1}{d}+\left(\frac{n-1}{2 n}\right) \frac{1}{d^{3}}+\left(\frac{2 n-5}{2 n}\right) \frac{1}{d^{4}}+\left(3-\frac{99}{8 n}+\frac{5}{8 n^{2}}\right) \frac{1}{d^{5}}+\mathrm{O}\left(d^{-6}\right) .
$$

Taking the limit $n \rightarrow \infty$, one recovers the results obtained by Blease (1977). So in this case the $n$-tree approximation method gives the right $1 / d$ expansion.

\subsection{The percolation problem in dimension $d=2$}

To further test the validity of the $n$-tree approximation method, we tried to calculate the ground-state energy per unit length, $E(p)$, for the distribution (78) in dimension two. A direct Monte Carlo simulation done on polymers of length $10^{6}$ leads to $E(0.1)=0.5537 \pm 0.0005$ and $E(0.2)=0.3719 \pm 0.0005$.

By calculating the prediction, $E_{n}(p)$, of the $n$-tree approximation of this problem, we have obtained the results shown in figure 6 for $1 \leqslant n \leqslant 8$. When plotted against $n^{-1 / 3}$, the results converge linearly to the results of the simulation. So here again, the $n$-tree approximation seems, in the limit $n \rightarrow \infty$, to converge to the right answer. There is probably a way of relating the convergence rate to the known exponents $\omega=\frac{1}{3}$ and $\nu=\frac{2}{3}$, in dimension $d=1+1$, but we were unable to find it.

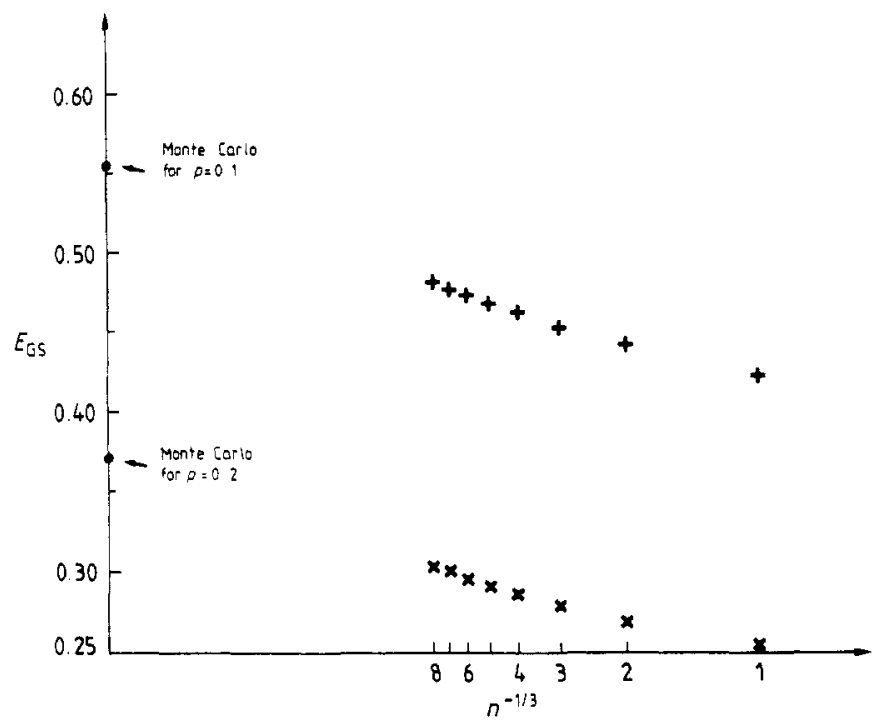

Figure 6. The $n$-tree approximation for the ground-state energy of the percolation problem in dimension two. Approximations are shown for $p=0.1(t)$ and $p=0.2(\times)$.

\subsection{The simplex problem}

Another test we considered for justifying the $n$-tree approximation was to study a simplified model of directed polymers in a random medium, the simplex model (see figure 7). The lattice in the transverse direction is a simplex and at each step along the preferred direction the polymer is allowed to jump from any corner of the simplex to any other corner. 


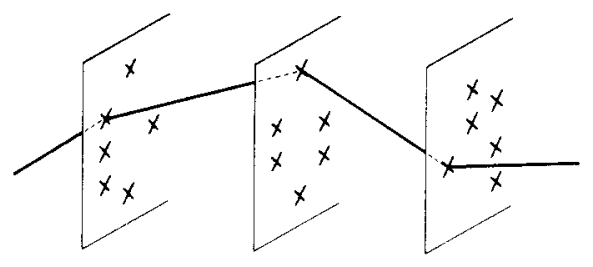

Figure 7. The simplex model.

So in the simplex model the lattice consists of $L$ planes with $K$ points on each plane. Each point of a given plane is connected to all points of the previous and next plane. Therefore there are $K^{2}$ links between two consecutive planes. As always, on each link between two planes one chooses a random energy, $\varepsilon$, according to a given distribution $\rho(\varepsilon)$. A directed walk is a walk which visits a single site in each plane, so the energy, $E_{\mathrm{w}}$, of a walk of $L$ steps is the sum of the energies of the bonds visited by the walk.

For this problem one can write the following recursion relation for the partition function:

$$
Z_{L+1}(i)=\sum_{j=1}^{K} \exp \left[-\varepsilon_{i j}(L) / T\right] Z_{L}(j)
$$

where $Z_{L}(i)$ is the partition function of the walks which reach the $i$ th point on plane $L$. So, the calculation of the free energy is the same as finding the largest Lyapounov exponent of a product of $K \times K$ matrices, the elements of which are random, positive, independent and identically distributed. To our knowledge, this is a problem which has not yet been solved for a general distribution $\rho(\varepsilon)$.

We were only able to solve this problem in the particular case of zero temperature, large $K$, and the percolation distribution

$$
\rho(\varepsilon)=p \delta(\varepsilon)+(1-p) \delta(1-\varepsilon) .
$$

For large $K$ it is convenient to use, instead of the parameter $p$ (in (85)), the parameter $x$ related to $p$ by

$$
p=K^{-x} \text {. }
$$

Let us first show that the ground-state energy per unit length, $E$, is given by

$$
E=\left(1+\left[\frac{1}{x-1}\right]\right)^{-1} \quad x \geqslant 1
$$

where in (87) the square brackets denote the integer part of the argument. It is possible to derive this result by writing a Master equation for the probability, $P_{L}\left(E_{1}, \ldots, E_{i}, \ldots, E_{K}\right)$ that the ground-state energies of the walks of length $L$ which reach the points $1, \ldots, i, \ldots, K$ of plane $L$ are $E_{1}, \ldots, E_{i}, \ldots, E_{K}$. This Master equation can be solved using the fact that the differences in energy $\left|E_{i}-E_{j}\right|$ can only be 0 or 1 , leading to (87) for large $K$. We shall only present here a simple argument which leads to $(87)$.

It is easy to calculate the typical number of points in plane $L=m$ that are connected by a path of zero energy to the plane $L=0$. This typical number is $K^{1+m(1-x)}$. As long as $1+m(1-x)$ is positive, there is a path of zero energy from plane 0 to $m$. So if $m$ is the largest integer such that

$$
1+m(1-x)>0 \quad \text { and } \quad 1+(m+1)(1-x)<0
$$


the minimum energy to go from plane 0 to plane $m+1$ is 1 . Therefore for large $L$ the ground-state energy is $L /(m+1)$ where $m$ satisfies (88), and so one gets (87).

Let us now present the $n$-tree approximation for this simplex problem. As in section 3 , the ground-state energy $E_{n}$ of the $n$th approximation will be given by

$$
E_{n}=-\left.\min _{\gamma} G_{n}(\gamma)\right|_{T=0}
$$

So we only need to determine the large- $K$ behaviour of $G_{n}(\gamma)$. Clearly, from (13),

$$
\begin{aligned}
G_{1}(\gamma) & =\frac{1}{\gamma} \log \left\{K\left[K^{-x}+\left(1-K^{-x}\right) \mathrm{e}^{-\gamma}\right]\right\} \\
& \simeq \frac{1}{\gamma} \log \left(K^{1-x}+K \mathrm{e}^{-\gamma}\right)
\end{aligned}
$$

so one has for large $K$

$$
E_{1}= \begin{cases}(x-1) / x & x \geqslant 1 \\ 0 & x \leqslant 1\end{cases}
$$

Going to the 2-tree approximation one can show that for large $K$

$$
G_{2}(y) \simeq \frac{1}{2 \gamma} \log \left(K^{2-2 x}+2 K^{2-x} \mathrm{e}^{-\gamma}+K \mathrm{e}^{-2 \gamma}\right) .
$$

This can be understood because the average number of points which can be reached after two steps starting from a given point behaves like: $K^{2-2 x}$ for energy $0, K^{2-x}$ for energy 1 and $K$ for energy 2. Minimising over $\gamma$ (equation (89)), one finds that for large $K$

$$
E_{2}= \begin{cases}0 & x \leqslant 1 \\ (x-1) / x & 1 \leqslant x \leqslant 2 \\ (2 x-3) / 2(x-1) & 2 \leqslant x .\end{cases}
$$

Already with this 2-tree approximation, we see an improvement toward the true answer (87).

We did not find a simple way of solving the $n$-tree approximation for arbitrary $x$. However, for $x>2$, we could generalise for arbitrary $n$ the reasoning which led to the expression of $G_{2}(\gamma)$. For example, for odd $n$, one gets for $x>2$

$G_{n}(\gamma)=\frac{1}{n \gamma} \log \left(\sum_{m=0}^{(n+1) / 2} b_{m} K^{n-n x+m x} \mathrm{e}^{-m \gamma}+\sum_{m=(n+3) / 2}^{n} b_{m} K^{2 n+1-2 m-n x+m x} \mathrm{e}^{-m \gamma}\right)$

where $b_{m}$ are constants independent of $K$.

This leads to

$$
E_{n}= \begin{cases}1-1 / n(x-2) & \text { for } x>2 n /(n-1) \\ (x-1) / x & \text { for } 2 n /(n-1)>x>2\end{cases}
$$

which converges to the exact result $E=1$ (see (87)) in the limit $n \rightarrow \infty$.

Similarly for even $n$ and $x>2$, one gets

$$
E_{n}= \begin{cases}1-1 / n(x-2) & \text { for } x>(2 n-2) /(n-2) \\ (2 x-3) / 2(x-1) & \text { for }(2 n-2) /(n-2)>x>2 .\end{cases}
$$

So with this example we see that the limits $K \rightarrow \infty$ and $n \rightarrow \infty$ commute at least for $x>2$ and since $K$ plays a similar role to $d$ for the hypercubic lattice it supports the idea that these commute in general. 


\section{Conclusion}

In this paper we have developed a method of obtaining $1 / d$ expansions for the problem of directed polymers in a random medium, using the $n$-tree approximation. This consists of building a series of tree problems ( $n$-trees) which give the hypercubic lattice in the limit $n \rightarrow \infty$. For our results to be valid one needs the limits $n \rightarrow \infty$ and $d \rightarrow \infty$ to commute. At present we have no proof of this, but in several examples, which can be solved independently (see section 7), we found that this $n$-tree method gave the correct answer.

We have obtained here the $1 / d$ expansions of several quantities of interest for the directed polymer problem: free energy (42), specific heat (44), transition temperature (45), ground-state energy (46), transverse fluctuations (62) and overlaps (70). In contrast to the mean-field case, the low-temperature phase in finite dimension is no longer frozen. However, within our $1 / d$ expansions, we find that the distribution of overlaps remains broad and that the transverse fluctuations remain linear in the length $L$ of the polymer. This would be consistent with the idea that there exists a finite upper critical dimension above which the low-temperature phase has broken replica symmetry and the mean-field exponents.

It would be nice to see whether our results could be recovered by another approach which would not require the use of the $n$-tree approximation. It would also be interesting to know whether our $1 / d$ expansions have a finite radius of convergence which could be identified with a finite upper critical dimension, or have a zero radius of convergence as has been noted for other 1/d expansions (Fisher and Singh 1989). This would require pushing our diagrammatic expansion further.

We think that the method presented here could be easily extended to other situations: finding the free energy as a function of the transverse displacement, site disorder rather than bond disorder. It should also be possible to extend it to other directed models and to growth models.

Lastly, in section 7, we introduced the simplex model which is, in spirit, in the directed polymer case, the equivalent of the Sherrington-Kirkpatrick (1975) model of spin glasses. This problem can be reduced to the calculation of the Lyapounov exponents for products of $K \times K$ random matrices, the elements of which are random, positive, independent and identically distributed. It would be nice to find a way of expanding the free energy of this model for large $K$.

\section{Acknowledgments}

We would like to thank H Spohn, J Vannimenus and J Yedidia for useful discussions. JC acknowledges support from SERC.

\section{Appendix 1. Solution of the tree problem using replicas}

In this appendix we derive the solution of the problem of directed polymers in a random medium on a tree using replicas. We also show how one can obtain the overlaps, $q_{2}$, using this method, and derive $(52 b)$.

For generality, consider a tree with branching ratio $K$. Out of the $K$ branches suppose that $k_{1}$ have random energies chosen according to the distribution $\rho_{1}(\varepsilon), k_{2}$ 
have random energies chosen according to $\rho_{2}(\varepsilon)$, etc $\ldots\left(\Sigma_{i} k_{i}=K\right)$. One can define a partition function for the problem, (2),

$$
Z_{L}=\sum_{*} \exp \left(-E_{w} / T\right)
$$

where the sum runs over all directed walks of length $L$ and $E_{w}$ is the total energy of the walk $w$ (see (1)).

The free energy will be calculated using the replica trick (Sherrington and Kirkpatrick 1975)

$$
\lim _{L \rightarrow \infty} \lim _{n \rightarrow 0} \frac{\log \left\langle Z_{L}^{n}\right\rangle}{n L}=\lim _{L \rightarrow \infty} \frac{\left\langle\log Z_{L}\right\rangle}{L} .
$$

$\left\langle Z_{L}^{n}\right\rangle$ can be thought of as the average of the partition function for $n$ walks or replicas. We assume, following the Parisi scheme (Parisi 1980a, b) that the arrangements of the $n$ walks which give the leading contribution to $\left\langle Z_{L}^{n}\right\rangle$ for large $L$ are organised as follows:

(i) the $n$ walks remain together initially for a length $L q_{1}$;

(ii) the walks then split into $m_{1}$ groups of $n / m_{1}$ walks each and remain so for a length $L\left(q_{2}-q_{1}\right)$;

(iii) the walks then split into $m_{j}$ groups of $n / m_{j}$ walks each remaining so for a length $L\left(q_{j+1}-q_{j}\right)$;

(iv) the walks finally separate into $m_{M}=n$ individual walks a length $L$ from the starting point.

Therefore, one has

$$
\begin{aligned}
& q_{0}=0 \leqslant q_{1} \leqslant \ldots \leqslant q_{j} \leqslant \ldots \leqslant q_{M}=1 \\
& 1=m_{0} \leqslant m_{1} \leqslant \ldots \leqslant m_{j} \leqslant \ldots \leqslant m_{M}=n .
\end{aligned}
$$

Then for large $L,\left\langle Z_{L}^{n}\right\rangle$ is dominated by

$$
\left\langle\left(Z_{L}\right)^{n}\right\rangle \simeq \max _{\left\{q_{i}\right\}} \max _{\left\{m_{i}\right\}} \max _{\left\{l_{i j}\right\}} \prod_{j} \frac{m_{j} !}{\prod_{i} l_{i j} !} \prod_{i} k_{i}^{L\left(q_{i+1}-q_{i}\right) l_{i i}}\left(\int \rho_{i}(\varepsilon) \exp \left(-n \varepsilon / m_{j} T\right) \mathrm{d} \varepsilon\right)^{L\left(q_{j+1}-q_{i}\right) l_{i i}}
$$

where $l_{i j}$ branches of class $i$ are occupied by walks at the $j$ th level and therefore $m_{j}=\Sigma_{i} l_{i j}$.

Taking the maximum over the $l_{i j}$, one gets

$$
\left\langle\left(Z_{L}\right)^{n}\right\rangle=\max _{\left\{q_{j}\right\}} \max _{\left\{m_{,}\right\}} \prod_{j} \exp \left[L\left(q_{j+1}-q_{j}\right) m_{j} \log \left(\sum_{i} k_{i} \int \rho_{i}(\varepsilon) \exp \left(-n \varepsilon / m_{j} T\right) \mathrm{d} \varepsilon\right)\right] \text {. }
$$

To evaluate $\left\langle Z_{L}^{n}\right\rangle$ for large $L$ one has to find the set $\left\{q_{j}\right\},\left\{m_{j}\right\}$ which maximises the exponent in (A1.5). In the limit $n \rightarrow 0$, required in (A1.2), following Parisi (Parisi $1980 \mathrm{a}, \mathrm{b})$, we invert the inequality $(\mathrm{Al} .3 \mathrm{~b})$ to give

$$
1=m_{0} \geqslant m_{1} \geqslant \ldots \geqslant m_{j} \ldots \geqslant m_{M}=n=0
$$

and take the limit $M \rightarrow \infty$ by defining a function, $x\left(q_{j}\right)$, such that

$$
m_{j}=\frac{n}{x\left(q_{j}\right)}
$$

with $q_{j}$ becoming a variable continuous on the interval $[0,1]$ and $0<x(q)<1$. Then using (A1.2) and (A1.5) one obtains

$$
\lim _{L \rightarrow \infty} \frac{1}{L}\left\langle\log Z_{L}\right\rangle=\operatorname{extr}_{x(q)} \int_{0}^{1} \frac{d q}{x(q)} \log \left(\sum_{i} k_{i} \int \rho_{i}(\varepsilon) \exp (-\varepsilon x(q) / T) \mathrm{d} \varepsilon\right) .
$$


As there is no explicit $q$ dependence in (A1.8), $x(q)$ must be a constant. Hence one finds that

$$
x(q)_{\text {optimum }}= \begin{cases}1 & T \geqslant T_{\mathrm{c}} \\ T \gamma_{\min }=T / T_{\mathrm{c}} & T \leqslant T_{\mathrm{c}}\end{cases}
$$

where $\gamma_{\min }$ is defined by

$$
\left.\frac{\mathrm{d} G(\gamma)}{\mathrm{d} \gamma}\right|_{\gamma_{\min }}=0
$$

with

$$
G(\gamma)=\frac{1}{\gamma} \log \left(\sum_{i} k_{i} \int \rho_{i}(\varepsilon) \mathrm{e}^{-\varepsilon \gamma} \mathrm{d} \varepsilon\right) .
$$

(It is easy to show that $\gamma G(\gamma)$ is a convex function of $\gamma$, and one can therefore show that (A1.10) has a unique solution.) So one can conclude from (A1.8) and (A1.9) that

$$
\lim _{L \rightarrow \infty} \frac{1}{L}\left\langle\log Z_{L}\right\rangle= \begin{cases}\frac{1}{T} G(1 / T) & T \geqslant T_{\mathrm{c}} \\ \frac{1}{T} G\left(\gamma_{\min }\right) & T \leqslant T_{\mathrm{c}} .\end{cases}
$$

This reduces to the result of section 2 (equations (13), (15)), derived using the travelling wave analogy, when one considers the case of a single distribution of disorder, $\rho(\varepsilon)$ (i.e. $k_{1}=K$ and $\rho_{1}(\varepsilon)=\rho(\varepsilon)$ ). For clarity we shall now restrict ourselves to this problem.

From this replica calculation one can determine the overlaps. These are defined in section 2 (equations (17), (18)). The probability distribution of the overlaps, $q_{2}$, between two walks can be calculated using the identification (Parisi 1983, Mezard et al 1984)

$$
P_{2}(q)=\frac{\mathrm{d} x(q)}{\mathrm{d} q}
$$

Hence, from (A1.9), one can see that for $T \leqslant T_{c}, P_{2}(q)$ is composed of two delta functions

$$
P_{2}(q)=T \gamma_{\min } \delta(q)+\left(1-T \gamma_{\min }\right) \delta(q-1) \quad T \leqslant T_{\mathrm{c}} .
$$

Above $T_{\mathrm{c}}, P_{2}(q)$ is a single delta function at $q=0$

$$
P_{2}(q)=\delta(q) \quad T \geqslant T_{\mathrm{c}} \text {. }
$$

In the low-temperature phase $P_{2}(q)$ is not a single delta function, $q_{2}$ is not self-averaging and one can conclude that the phase has broken replica symmetry. This is analogous to the overlaps in the low-temperature phase of the mean-field spin glass (Mezard et al 1987).

One can, in addition, extract some information about the valley structure of the energy landscape from (A1.14). The replica symmetry breaking indicates a many-valley energy landscape. Assume one has a certain number of valleys, each valley $\alpha$ having a weight $\omega_{\alpha}$. If two walks fall in the same valley they have an overlap of unity and if they are in different valleys they have a zero overlap. Hence from (A1.14) it follows that

$$
\sum_{\alpha} \omega_{\alpha}^{2}=1-T \gamma_{\min } \quad T \leqslant T_{c} .
$$

This is the result $(52 b)$ used in section 4 . 


\section{Appendix 2. Derivation of equation (65)}

The $n$-tree approximation to the $d$-dimensional lattice is a tree structure consisting of branches of a number of types. For example, the 2-tree consists of two types of branches, those which are formed by only one path and those which are made up of two paths (see figure $2(b)$ ). In this appendix we show how one can calculate the fraction of each type of branch used by a typical walk on an $n$-tree. This quantity is needed in the calculation of the overlaps in section 5 .

To see how one can evaluate these fractions, let us consider the simpler problem of directed walks on a tree when one has a discrete distribution of energies. Suppose that the probability of choosing a bond with energy $\varepsilon_{i}$ is given by $\rho_{i}$, the $\varepsilon_{i}$ forming a discrete set $\left\{\varepsilon_{i}\right\}$

$$
\rho(\varepsilon)=\sum_{i} \rho_{i} \delta\left(\varepsilon-\varepsilon_{i}\right)
$$

The free energy of the system per unit length, $L$, is then given by (15)

$$
F(T)= \begin{cases}-G\left(\gamma_{\min }\right) & T \leqslant T_{\mathrm{c}} \\ -G(1 / T) & T \geqslant T_{\mathrm{c}}\end{cases}
$$

where

$$
G(\gamma)=\frac{1}{\gamma} \log \left(\sum_{i} \rho_{i} \exp \left(-\gamma \varepsilon_{i}\right)\right)
$$

and $\gamma_{\min }$ is the unique solution of

$$
\left.\frac{\mathrm{d} G(\gamma)}{\mathrm{d} \gamma}\right|_{\gamma_{\min }}=0 \text {. }
$$

Let $N_{i}$ denote the number of branches of type $i$ in the walks. One can then write the partition function $Z_{L}$ as

$$
Z_{L}=\sum_{N_{1}=0}^{L} z_{L}\left(N_{1}\right) \exp \left(-\varepsilon_{1} N_{1} / T\right)
$$

where $z_{L}\left(N_{1}\right)$ is the partial partition function for all walks of length $L$ containing exactly $N_{1}$ steps on branches of type 1 . Our aim is to calculate the average number of steps on branches of type 1

$$
\left\langle\bar{N}_{1}\right\rangle=\left\langle Z_{L}^{-1} \sum_{N_{1}=0}^{L} N_{1} z_{L}\left(N_{1}\right) \exp \left(-\varepsilon_{1} N_{1} / T\right)\right\rangle
$$

where denotes a thermal average. Comparing (A2.6) and (A2.5) we see that the average of $N_{1}$ can be obtained as a derivative of the free energy

$$
\left\langle\bar{N}_{1}\right\rangle=-T \frac{\partial}{\partial \varepsilon_{1}}\left\langle\log Z_{L}\right\rangle
$$

and so, from (A2.2)-(A2.4) we see that

$$
\left\langle\bar{N}_{1} / L\right\rangle=\frac{\partial F}{\partial \varepsilon_{1}}=\frac{\rho_{1} \exp \left(-\gamma \varepsilon_{1}\right)}{\Sigma_{i} \rho_{i} \exp \left(-\gamma \varepsilon_{i}\right)}
$$

where $\gamma=\gamma_{\min }$ for $T \leqslant T_{\mathrm{c}}$ and $\gamma=1 / T$ for $T \geqslant T_{\mathrm{c}}$. 
This idea can be extended to continuous distributions and used to calculate the average fraction of each type of branch used by walks on an $n$-tree. As the simplest example we shall take the 2 -tree, although the extension to the $n$-tree is easy. From (32) and (36) the free energy per unit length of the 2-tree is given in the low-temperature phase by

$$
\begin{aligned}
F_{2}(T)=-\frac{1}{2 \gamma_{\min }} & \log \left[d\left\langle\exp \left(-\varepsilon \gamma_{\min }\right)\right\rangle^{2}+\frac{1}{2} d(d-1)\right. \\
\times & \left\langle\left\{\exp \left[-\left(\varepsilon_{1}+\varepsilon_{2}\right) / T\right]+\exp \left[-\left(\varepsilon_{3}+\varepsilon_{4}\right) / T\right]\right\}^{\left.\left.T \gamma_{\min }\right\rangle\right]}\right.
\end{aligned}
$$

where $\gamma_{\min }$ is defined by (32) and (36). To calculate the overlap for the 2-tree (see section 5) one needs to know the average fraction of branches used in a walk on the 2-tree which consist of two paths with energies $\varepsilon_{1}+\varepsilon_{2}$ and $\varepsilon_{3}+\varepsilon_{4}$ on the constituent links. Extending (A2.8) to continuous distributions and effective bonds, one can see from (A2.9) that this fraction is given in the low-temperature phase by

$$
\frac{\frac{1}{2} d(d-1)\left(\exp \left[-\left(\varepsilon_{1}+\varepsilon_{2}\right) / T\right]+\exp \left[-\left(\varepsilon_{3}+\varepsilon_{4}\right) / T\right]\right)^{T \gamma_{\min }} \rho\left(\varepsilon_{1}\right) \rho\left(\varepsilon_{2}\right) \rho\left(\varepsilon_{3}\right) \rho\left(\varepsilon_{4}\right)}{d\left(\exp \left(-\varepsilon \gamma_{\min }\right)\right\rangle^{2}+\frac{1}{2} d(d-1)\left\langle\left\{\exp \left[-\left(\varepsilon_{1}+\varepsilon_{2}\right) / T\right]+\exp \left[-\left(\varepsilon_{3}+\varepsilon_{4}\right) / T\right]\right\}^{\left.T \gamma_{\min }\right\rangle}\right.} .
$$

This is the result quoted in (65). This method can easily be extended to calculate the average fraction of its length spent by a walk on any type of branch on the $n$-tree.

\section{References}

Blease J 1977 J. Phys. C: Solid State Phys. 10925

Bramson M 1983 Convergence of solutions of the Kolmogorov equation to travelling waves Memories of the American Mathematical Society 285

Bray A J and Moore M A 1979 J. Phys. C: Solid State Phys. 1279

Cook J and Derrida B 1989a Europhys. Lett. 10195

1989b J. Stat. Phys. 5789

de Dominicis C and Kondor I 1985 J. Physique Lett. $46 \mathrm{~L} 1037$

Derrida B and Gardner E 1986 J. Phys. C: Solid State Phys. 195783

Derrida B and Spohn H 1988 J. Stat. Phys. 51817

Dhar D 1988 Phys. Lett. 130A 308

Fisher M E and Singh R R P 1989 Disorder in Physical Systems ed G Grimmett and D J A Welsh, to appear Forster D, Nelson D R and Stephen M J 1977 Phys. Rev. A 16732

Halpin-Healy T 1989 Phys. Rev. Lett. 62442

Hammersley J M 1974 Ann. Prob. 2652

Huse D A and Fisher D S 1987 J. Phys. A: Marh. Gen. 20 L997

1988 Phys. Rev. B 38386

Huse D A, Henley C L and Fisher D S 1985 Phys. Rev. Lett. 552924

Imbrie J Z and Spencer T 1988 J. Stat. Phys, 52609

Kardar M 1987 J. Appl. Phys. 613601

Kardar M, Parisi G and Zhang Y C 1986 Phys. Rev. Lett. 56889

Kardar M and Zhang Y C 1987 Phys. Rev. Lett. 582087

Kim J M and Kosterlitz J M 1989 Phys. Rev. Lett. 622289

Krug J 1987 Phys. Rev. A 365465

Krug J and Spohn H 1988 Phys. Rev. A 384271

McKane A J and Moore M A 1988 Phys. Rev. Lett. 60527

Mezard M, Parisi G, Sourlas N, Toulouse G and Virasoro M 1984 J. Physique 45843

Mezard M, Parisi G and Virasoro M 1987 Spin Glass Theory and Beyond (Singapore: World Scientific)

Nattermann T and Renz W 1988 Phys. Rev. B 385184 
Parisi G 1980a J. Phys. A: Math. Gen. 131101

- 1980 b J. Phys. A: Marh. Gen. 131887

1983 Phys. Rev. Lett. 501946

Renz W 1989 to be published

Sherrington D and Kirkpatrick S 1975 Phys. Rev. Lett. 321792

Sourlas N 1986 Europhys. Lett. 1189

Wolf D E and Kertész J 1987 Europhys. Lett. 4651

Zhang Y C 1987 Phys, Rev. Lett. 592125 